\title{
Hybrid meshless/displacement discontinuity method for FGM Reissner's plate with cracks
}

\author{
H. Zheng ${ }^{1}$, J. Sladek ${ }^{2}$, V. Sladek ${ }^{2}$, S.K. Wang ${ }^{3}$, P.H. Wen ${ }^{3 *}$ \\ ${ }^{1}$ School of Civil Engineering, Nanchang University, Nanchang, China \\ ${ }^{2}$ Institute of Construction and Architecture, Slovak Academy of Sciences, Bratislava, Slovakia \\ ${ }^{3}$ School of Engineering and Materials Science, Queen Mary University of London, London, UK
}

\begin{abstract}
Growing applications of non-homogenous media in engineering structures require the application of powerful computational tools. A novel hybrid Meshless Displacement Discontinuity Method (MDDM) for cracked Reissner's plate in Functionally Graded Materials (FGMs) is presented in this paper. The fundamental solutions of slope and deflection discontinuity for an isotropic homogenous media are chosen as a part of general solutions to create the gaps between the crack surfaces. The governing equation is satisfied by using the meshless methods such as the Meshless Local Petrov-Galerkin (MLPG) and the Point Collocation Method (PCM) with Lagrange series interpolation and mapping technique. The Stress Intensity Factors (SIFs) are evaluated analytically with the Chebyshev polynomials. The accuracy is verified by comparison of numerical and analytical results.
\end{abstract}

Key words: Functionally graded materials, Kirchhoff and Reissner plate theories, boundary element method, displacement discontinuity method, meshless local Petrov-Galerkin method, stress intensity factors.

*Corresponding author: p.h.wen@qmul.ac.uk 


\section{Introduction}

Plate and shells are main components in aerospace structures. There are two major theories of plates and shells in engineering, namely the Kirchhoff thin plate theory and the Reissner moderately thick plate theory. The application of the Finite Element Method (FEM) to the Kirchhoff plate and Reissner plate was reported by Zienkiewicz and Taylor [1]. More recent researches on the fracture analysis of plates and shells can be found in [2][3][4]. The application of the Boundary Element Method (BEM) to the plate was first proposed by Jaswon et al [5], based on the bi-harmonic analysis with two unknown boundary values i.e. the deflection and normal slope. The derivation of the boundary integral equation and the fundamental solution for the Reissner's plate theory was reported by Vander Weeën [6]. Karam and Telles [7] have shown that the Reissner's plate model can be applied to the Kirchhoff thin plate too. Rashed et al [8,9] derived the traction integral equation for the Reissner's plate theory to solve the boundary value problems. Dirgantara and Aliabadi [10,11,12] extended the application of dual BEM to the fracture analysis of Reissner's plate successfully. Fracture analysis of curved stiffened panels, crack growth analysis for multi-layered airframe structures and non-linear large deformation analysis of Reissner's plate by Boundary Element Method were reported by Wen et al $[13,14,15]$. Recent developments in boundary element method for plate bending analysis can be found in the book by Aliabadi [16].

Recently, the meshless methods such as Element-free Galerkin method and Point collocation method were developed and have attracted huge attention for solving boundary value problems. The Diffuse element method was reported by Nayroles et al [17], the Elementfree Galerkin method and the reproducing kernel particle methods were proposed by Belyschko et al [18] and Liu et al [19]. Atluri and his colleagues presented a set of meshless methods, named as Meshless Local Petrov-Galerkin formulations (MLPG), for solving partial differential equations [20,21 and 22] with the Moving-Least Square (MLS) approximation. The MLPG was reported to provide a rational basis for constructing meshless methods with greater applicability. In addition, the Local integral equation method with the MLS and polynomial radial function was proposed by Sladek et al $[23,24,25]$. A comprehensive review of the meshless methods can be found in Atluri [24] and Liu [26].

It is well known that the Method of Fundamental Solutions (MFS) is a simple and efficient technique to solve the partial differential equations numerically. However, there are just a few 
works on modeling linear elastic fracture mechanics problems using the MFS. The Green's function approach including the adopted numerical Green's function (NGF) avoids boundary elements over the crack surfaces, since the fundamental solution removes boundary integration there. Guimarães and Telles [27,28] developed the numerical Green's function formulation used as the fundamental solution for Reissner's plate for stress intensity factor computations. Sladek et al $[29,30,31]$ developed a meshless method based on the local Petrov-Galerkin weak-form to solve thermal problems of orthotropic thick plates with material properties continuously varying through the plate thickness. Wen and Hon [32] extended meshless method to nonlinear analysis of the Reissner's plate.

Regarding the functionally graded materials, it is clear that the mechanical properties of the material vary with the coordinates. It leads to significant difficulty in obtaining solutions of the complex partial differential equations in anisotropic and non-homogeneous media. Although FEM, BEM and meshless methods can be applied to find the numerical solutions, they have different difficulties. The main issue for the FEM is the accuracy due to the discontinuity of the stresses between elements. The availability of the fundamental solution is essential for the BEM and the convergence by using the meshless methods needs more attention in computations.

For 2D/3D elasticity, the dual boundary element method with a single region technique for the crack growth analysis was demonstrated by Portela et al [33] and by Mi and Aliabadi [34]. Displacement Discontinuity Method (DDM) was reported by Crouch [35]. Wen et al [36,37] extended the DDM to fracture static/dynamic 2D/3D problems successfully. The hybrid MDDM is a novel investigation of the Stress Intensity Factor (SIFs) at the tip for the Reissner's plate theory for non-homogenous media. The motivation of the MDDM proposed recently by $\mathrm{Li}$ et al [38] inherits the advantages from the boundary element method and meshless method. The general solution consists of two parts: (a) a solution with an embedded crack in an infinite plate of isotropic homogenous material; (b) a solution for a finite non-homogenous material without crack. The first one is obtained by using boundary integral equation method (displacement discontinuity), in order to create the gap between crack surfaces and the second part is determined by the meshless method, in order to consider the governing equation in the domain. For the first solution, the problem is transformed to solve a set of singular integral equations with hyper and strong singularities. Highly accurate numerical solutions can be obtained by using the Chebyshev polynomials. An analytical treatment enables us to achieve the closed 
form solutions of SIFs at the tip in functionally graded materials. In this paper, the formulations with the MDDM for the cracked Reissner plate theory of FGM are firstly derived with distributed dislocation of the slopes and deflection on the crack surface (discontinuous field in infinite plate) and the nodal displacements in the field and on the boundary as well (continuous field). Finally, several numerical examples are presented to illustrate the applicability of the MDDM and the comparative analysis has been carried out for the cracked FGM plates with different approaches.

\section{Embedded crack in infinite homogenous plate}

In linear elasticity, functionally graded materials are designed to exhibit a particular spatial variation of their properties. It will be assumed in-plane gradation of Young's modulus $E(\mathbf{x})$, in this paper. For both homogenous and non-homogenous materials, the stress resultant-strain relationship for plate bending are given by Reissner [39]

$$
\begin{aligned}
& M_{\alpha \beta}=\frac{(1-v) D}{2}\left(w_{\alpha, \beta}+w_{\beta, \alpha}+\frac{2 v}{1-v} w_{\gamma, \gamma} \delta_{\alpha \beta}\right), \\
& Q_{\alpha}=C\left(w_{\alpha}+w_{3, \alpha}\right),
\end{aligned}
$$

where $D=E(\mathbf{x}) h^{3} / 12\left(1-v^{2}\right)$ is the bending stiffness of the plate, $E(\mathbf{x})$ is Young's modulus, $v$ is Poisson ratio and defined as a constant, $M_{\alpha \beta}$ are the bending and twisting moments per unit length, $Q_{\alpha}$ is the shearing force per unit length, $C=D(1-v) \lambda^{2} / 2$ is the shear stiffness, $\lambda=\sqrt{10} / h$ is shear factor, $h$ denotes the thickness of the plate, $w_{\alpha}$ is the slope, $w_{3}$ is the outof-plane deflection normal to the middle surface of the plate, $\delta_{\alpha \beta}$ is the Kronecker delta and Latin indices vary from 1 to 2 . Bearing in mind the definition of the bending stress-couples $M_{\alpha \beta}$, shear stress-resultants $Q_{\alpha}$ and supposing the quadratic distribution of shear stresses as well as boundary conditions on the plate surfaces, one can write the following relationships

$$
\sigma_{\alpha \beta}=\frac{12 x_{3}}{h^{3}} M_{\alpha \beta}, \quad \sigma_{\alpha 3}=\frac{3}{2 h}\left[1-\left(\frac{2 x_{3}}{h}\right)^{2}\right] Q_{\alpha}, \quad \sigma_{33}=\frac{2 x_{3}}{h}\left[3-\left(\frac{2 x_{3}}{h}\right)^{2}\right] \frac{q}{4},
$$

with $\sigma_{33}= \pm q / 2$ being the transversal loading on the plate surfaces $x_{3}= \pm h / 2$.

The equations of equilibrium can be written as 


$$
M_{\alpha \beta, \beta}-Q_{\alpha}=0 \quad \text { and } \quad Q_{\alpha, \alpha}+q=0
$$

in which $q$ denotes the pressure load on the plate. Differentiations of Eq.(1) yield

$$
\begin{aligned}
& M_{\alpha \beta, \beta}=\frac{(1-v) D w_{\alpha, \beta \beta}}{2}+\frac{(1+v) D w_{\beta, \alpha \beta}}{2}+\frac{(1-v) D_{, \beta}}{2}\left(w_{\alpha, \beta}+w_{\beta, \alpha}+\frac{2 v}{1-v} w_{\gamma, \gamma} \delta_{\alpha \beta}\right), \\
& Q_{\alpha, \alpha}=C\left(w_{\alpha, \alpha}+w_{3, \beta \beta}\right)+C_{, \alpha}\left(w_{\alpha}+w_{3, \alpha}\right) .
\end{aligned}
$$

Substituting Eq.(4) into the equilibrium equations (3), we have

$$
\begin{aligned}
& \frac{(1-v) D w_{\alpha, \beta \beta}}{2}+\frac{(1+v) D w_{\beta, \alpha \beta}}{2}+\frac{D_{, \beta}}{D} M_{\alpha \beta}-C\left(w_{\alpha}+w_{3, \alpha}\right)=0, \\
& C\left(w_{\alpha, \alpha}+w_{3, \beta \beta}\right)+q+\frac{C_{, \alpha}}{C} Q_{\alpha}=0 .
\end{aligned}
$$

Considering homogenous media and applying the reciprocal theorem, we obtain the integral representations of slopes and deflection $[8,10]$ by .

$$
C(\mathbf{x}) w_{i}(\mathbf{x})=\int_{\Gamma}\left(W_{i j}^{*}\left(\mathbf{x}, \mathbf{x}^{\prime}\right) p_{j}\left(\mathbf{x}^{\prime}\right)-P_{i j}^{*}\left(\mathbf{x}, \mathbf{x}^{\prime}\right) w_{j}(\mathbf{x})\right) d \Gamma\left(\mathbf{x}^{\prime}\right)+\int_{\Omega} W_{i j}^{*}\left(\mathbf{x}, \mathbf{x}^{\prime}\right) q\left(\mathbf{x}^{\prime}\right) d \Omega\left(\mathbf{x}^{\prime}\right),
$$

where $p_{j}\left(\mathbf{x}^{\prime}\right)$ are the generalized tractions defined as

$$
p_{\alpha}\left(\mathbf{x}^{\prime}\right)=M_{\alpha \beta} n_{\alpha} \text { and } \quad p_{3}\left(\mathbf{x}^{\prime}\right)=Q_{\alpha} n_{\alpha}
$$

on the boundary contour $\Gamma$ of the domain $\Omega$ in the mid-plane of the plate. Furthermore, $n_{\alpha}$ are components of the unit outward normal vector on $\Gamma, \mathbf{x}$ and $\mathbf{x}^{\prime}$ are the source and field points respectively, $C(\mathbf{x})$ is a geometric coefficient at $\mathbf{x}$, which equals to either $\delta_{i j}$ or $0.5 \delta_{i j}$ for either an internal point or a point on a smooth part of the boundary, $W_{i j}^{*}\left(\mathbf{x}, \mathbf{x}^{\prime}\right)$ and $P_{i j}^{*}\left(\mathbf{x}, \mathbf{x}^{\prime}\right)$ are displacement and traction fundamental solutions [6] in an infinite domain and can be written, for plane stress circumstance, as

$$
\begin{aligned}
& W_{\alpha \beta}^{*}=\frac{1}{8 \pi D(1-v)}\left\{[8 B(z)-(1-v)(2 \ln z-1)] \delta_{\alpha \beta}-\left[8 A(z)+2(1-v) r_{, \alpha} r_{, \beta}\right]\right\}, \\
& W_{\alpha 3}^{*}=-W_{3 \alpha}^{*}=\frac{1}{8 \pi D}(2 \ln z-1) r r_{, \alpha}, \\
& W_{33}^{*}=\frac{1}{8 \pi D(1-v) \lambda^{2}}\left[(1-v) z^{2}(\ln z-1)-8 \ln z\right]
\end{aligned}
$$

and 


$$
\begin{aligned}
P_{\alpha \beta}^{*}= & \frac{-1}{4 \pi r}\left[\left(4 A(z)+2 z K_{1}(z)+1-v\right)\left(r_{, n} \delta_{\alpha \beta}+r_{, \beta} n_{\alpha}\right)+\right. \\
& \left.+(4 A(z)+1+v) r_{, \alpha} n_{\beta}-2\left(8 A(z)+2 z K_{1}(z)+1-v\right) r_{, \alpha} r_{, \beta} r_{, n}\right] \\
P_{\alpha 3}^{*}= & \frac{\lambda^{2}}{2 \pi}\left[B(z) n_{\alpha}-A(z) r_{, \alpha} r_{, n}\right], \\
P_{3 \alpha}^{*}= & \frac{-(1-v)}{8 \pi}\left[\left(2 \frac{1+v}{1-v} \ln z-1\right) n_{\alpha}+2 r_{, \alpha} r_{, n}\right], \\
P_{33}^{*}= & \frac{-1}{2 \pi r} r_{, n},
\end{aligned}
$$

where

$$
A(z)=K_{0}(z)+\frac{2}{z}\left[K_{1}(z)-\frac{1}{z}\right] \text { and } B(z)=K_{0}(z)+\frac{1}{z}\left[K_{1}(z)-\frac{1}{z}\right]
$$

in which $K_{0}(z)$ and $K_{1}(z)$ are modified Bessel functions of the second kind [6], $z=\lambda r$, $r=\sqrt{r_{\alpha} r_{\alpha}}$ is the distance from the source point to the field point with the components $r_{\alpha}=x_{\alpha}^{\prime}-x_{\alpha}$, the derivatives of $r$ are defined as $r_{, \alpha}=\partial r / \partial x_{\alpha}^{\prime}, r_{, n}=r_{, \alpha} n_{\alpha}$. Substituting Eq.(6) into Eq.(4) yields

$$
M_{\alpha \beta}(\mathbf{x})=\int_{\Gamma}\left(W_{\alpha \beta k}^{*}\left(\mathbf{x}, \mathbf{x}^{\prime}\right) p_{k}\left(\mathbf{x}^{\prime}\right)-D P_{\alpha \beta k}^{*}\left(\mathbf{x}, \mathbf{x}^{\prime}\right) w_{k}(\mathbf{x})\right) d \Gamma\left(\mathbf{x}^{\prime}\right)+\int_{\Omega} W_{\alpha \beta 3}^{*}\left(\mathbf{x}, \mathbf{x}^{\prime}\right) q\left(\mathbf{x}^{\prime}\right) d \Omega\left(\mathbf{x}^{\prime}\right)
$$

and

$$
Q_{\alpha}(\mathbf{x})=\int_{\Gamma}\left(W_{3 \alpha k}^{*}\left(\mathbf{x}, \mathbf{x}^{\prime}\right) p_{k}\left(\mathbf{x}^{\prime}\right)-D P_{3 \alpha k}^{*}\left(\mathbf{x}, \mathbf{x}^{\prime}\right) w_{k}(\mathbf{x})\right) d \Gamma\left(\mathbf{x}^{\prime}\right)+\int_{\Omega} W_{3 \alpha 3}^{*}\left(\mathbf{x}, \mathbf{x}^{\prime}\right) q\left(\mathbf{x}^{\prime}\right) d \Omega\left(\mathbf{x}^{\prime}\right),
$$

where the integral kernels are defined

$$
\begin{aligned}
W_{\alpha \beta \gamma}^{*}= & \frac{1}{4 \pi r}\left[-2\left(8 A(z)+2 z K_{1}(z)+1-v\right) r_{, \alpha} r_{, \beta} r_{, \gamma}+\left(4 A(z)+2 z K_{1}(z)+1-v\right)\left(\delta_{\beta \gamma} r_{, \alpha}+\delta_{\alpha \gamma} r_{, \beta}\right)+\right. \\
& \left.\quad+(4 A(z)+1+v) \delta_{\alpha \beta} r_{, \gamma}\right] \\
W_{\alpha \beta 3}^{*}= & \frac{-(1-v)}{8 \pi}\left[\left(2 \frac{1+v}{1-v} \ln z-1\right) \delta_{\alpha \beta}+2 r_{, \alpha} r_{, \beta}\right], \\
W_{3 \beta \gamma}^{*}= & \frac{\lambda^{2}}{2 \pi}\left[B(z) \delta_{\gamma \beta}-A(z) r_{, \beta} r_{, \gamma}\right], \\
W_{3 \beta 3}^{*}= & \frac{1}{2 \pi r} r_{, \beta},
\end{aligned}
$$

and 


$$
\begin{aligned}
& P_{\alpha \beta \gamma}^{*}=\frac{(1-v)}{4 \pi r^{2}}\left\{\left(4 A(z)+2 z K_{1}(z)+1-v\right)\left(\delta_{\gamma \alpha} n_{\beta}+\delta_{\gamma \beta} n_{\alpha}\right)\right. \\
& +(4 A(z)+1+3 v) \delta_{\alpha \beta} n_{\gamma}-\left(16 A(z)+6 z K_{1}(z)+z^{2} K_{0}(z)+2-2 v\right) \\
& \times\left[\left(n_{\alpha} r_{, \beta}+n_{\beta} r_{, \alpha}\right) r_{, \gamma}+\left(\delta_{\gamma \alpha} r_{, \beta}+\delta_{\gamma \beta} r_{, \alpha}\right) r_{, n}\right] \\
& -2\left(8 A(z)+2 z K_{1}(z)+1+v\right)\left(\delta_{\alpha \beta} r_{, \gamma} r_{, n}+n_{\gamma} r_{, \alpha} r_{, \beta}\right) \\
& \left.+4\left(24 A(z)+8 z K_{1}(z)+z^{2} K_{0}(z)+2-2 v\right) r_{, \alpha} r_{, \beta} r_{, \gamma} r_{, n}\right\} \text {, } \\
& P_{\alpha \beta 3}^{*}=\frac{(1-v) \lambda^{2}}{4 \pi r}\left[\left(2 A(z)+z K_{1}(z)\right)\left(r_{, \beta} n_{\alpha}+r_{, \alpha} n_{\beta}\right)\right. \\
& \left.-2\left(4 A(z)+z K_{1}(z)\right) r_{, \alpha} r_{, \beta} r_{, n}+2 A(z) \delta_{\alpha \beta} r_{, n}\right], \\
& P_{3 \beta \gamma}^{*}=\frac{-(1-v) \lambda^{2}}{4 \pi r}\left[\left(2 A(z)+z K_{1}(z)\right)\left(\delta_{\gamma \beta} r_{n}+r_{, \gamma} n_{\beta}\right)\right. \\
& \left.+2 A(z) n_{\gamma} r_{, \beta}-2\left(4 A(z)+z K_{1}(z)\right) r_{, \gamma} r_{, \beta} r_{, n}\right] \text {, } \\
& P_{3 \beta 3}^{*}=\frac{(1-v) \lambda^{2}}{4 \pi r^{2}}\left[\left(z^{2} B(z)+1\right) n_{\beta}-\left(z^{2} A(z)+2\right) r_{, \beta} r_{, n}\right] \text {. }
\end{aligned}
$$

Consider an embedded straight crack in an infinite plate with generalized traction over the crack boundary $p_{i}\left(\mathbf{x}^{+}\right)=-p_{i}\left(\mathbf{x}^{-}\right)$, the superscripts + and - indicate the upper and lower faces of the crack. Writing the integrals only over boundary $\Gamma^{-}\left(=\Gamma_{c}\right)$, the classical and hypersingular formulations in an infinite sheet, derived from Eq.(6) without pressure load $(q=0)$, are simplified as

$$
w_{i}(\mathbf{x})=\int_{\Gamma_{c}} P_{i j}^{*}\left(\mathbf{x}, \mathbf{x}_{c}\right) \psi_{j}\left(\mathbf{x}_{c}\right) d \Gamma\left(\mathbf{x}_{c}\right)
$$

where $\psi_{i}\left(\mathbf{x}_{c}\right)=w_{i}\left(\mathbf{x}_{c}^{+}\right)-w_{i}\left(\mathbf{x}_{c}^{-}\right)(i=1,2,3)$ is defined as a displacement discontinuity. Then, the stress resultants at the domain point in the case of homogenous material can be evaluated from Eqs. (10)-(11) as

$$
M_{\alpha \beta}(\mathbf{x})=D \int_{\Gamma_{c}} P_{\alpha \beta k}^{*}\left(\mathbf{x}, \mathbf{x}_{c}\right) \psi_{k}\left(\mathbf{x}_{c}\right) d \Gamma\left(\mathbf{x}_{c}\right)
$$

and

$$
Q_{\alpha}(\mathbf{x})=D \int_{\Gamma} P_{3 \alpha k}^{*}\left(\mathbf{x}, \mathbf{x}_{c}\right) \psi_{k}\left(\mathbf{x}_{c}\right) d \Gamma\left(\mathbf{x}_{c}\right) .
$$

Multiplying Eqs (15) and (16) by outward normal $n_{\beta}\left(\mathbf{x}^{-}\right)$, we obtain generalized tractions 
on the lower crack surface $\mathbf{x}^{-}$as

$$
p_{i}\left(\mathbf{x}^{-}\right)=n_{\beta}\left(\mathbf{x}^{-}\right) D \underset{\Gamma_{c}}{f_{i \beta k}} P^{*}\left(\mathbf{x}^{-}, \mathbf{x}_{c}\right) \psi_{k}\left(\mathbf{x}_{c}\right) d \Gamma\left(\mathbf{x}_{c}\right)
$$

where $f$ indicates the Hadamard principal value integral [6]. It is clear that the fundamental solution $P_{i j}^{*}$ involves strong singularity of $O(1 / r)$ and can be rewritten as

$$
P_{i j}^{*}=\frac{f_{i j}}{r}+O(\ln r)
$$

where

$$
f_{\alpha \beta}=-\frac{1}{4 \pi}\left[(1-v) r_{, \beta} n_{\alpha}+(1+v) r_{, \alpha} n_{\beta}\right], \quad f_{\alpha 3}=f_{3 \alpha}=f_{33}=0
$$

On the straight crack surface, the fundamental solution $P_{i \beta k}^{*}$ has hyper-singularity of $O\left(1 / r^{2}\right)$ as

$$
P_{i j k}^{*}=\frac{g_{i j k}}{r^{2}}+\frac{f_{i j k}}{r}+O(\ln r)
$$

where

$$
\begin{aligned}
g_{\alpha \beta \gamma}= & \frac{(1-v)}{4 \pi}\left[(1-v)\left(\delta_{\gamma \alpha} n_{\beta}+\delta_{\gamma \beta} n_{\alpha}\right)-(1-3 v) \delta_{\alpha \beta} n_{\gamma},\right. \\
& \left.+2 v\left(n_{\alpha} r_{, \beta}+n_{\beta} r_{, \alpha}\right) r_{, \gamma}+2(1-v) n_{\gamma} r_{, \alpha} r_{, \beta}\right] \\
g_{3 \beta 3}= & \frac{(1-v) \lambda^{2}}{4 \pi} n_{\beta}, \\
f_{\alpha \beta k}= & f_{3 \beta 3}=0, \quad f_{3 \beta \gamma}=\frac{(1-v) \lambda^{2}}{4 \pi} n_{\gamma} r_{, \beta} .
\end{aligned}
$$

\section{Displacement discontinuity method for Reissner's plate}

Consider a straight crack of length $2 a$ in a isotropic homogenous plate, as shown in Figure 1 (in this case $n_{1}\left(\mathbf{x}^{-}\right)=0, n_{2}\left(\mathbf{x}^{-}\right)=1$ on $\Gamma_{c}=\Gamma^{-} \ni \mathbf{x}^{-}$with $\left.\left(x_{1}^{-}, x_{2}^{-}\right)=(x, 0)\right)$, therefore, the integral equation (17) can be rewritten as

$$
f_{-a}^{a} P_{i 2 k}^{*}(x, \xi) \psi_{k}(\xi) d \xi=-\frac{p_{i}(x)}{D} .
$$




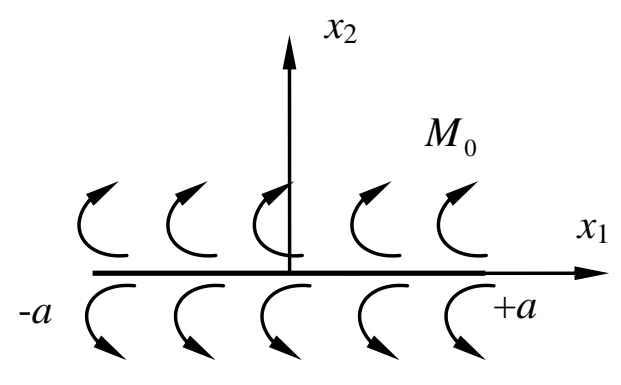

Figure 1. Embedded crack in an infinite plate subjected to bending moment $M_{0}$.

For mode I (opening) fracture, the integral equation above gives

$$
f_{-a}^{a} P_{222}^{*}(x, \xi) \psi_{2}(\xi) d x=-\frac{\bar{M}(x)}{D},
$$

because $P_{22 k}^{*}(x, \xi): \delta_{k 2}$, since $n_{i}=\delta_{i 2}$ and $r_{, 2}=0, n_{i} r_{, i}=0$ on $\Gamma^{-}$. However, model II $\psi_{1}(\xi)$ (sliding) and mode III $\psi_{3}(\xi)$ (tearing) fractures are coupled in the following equations

$$
\begin{aligned}
& f_{-a}^{a}\left[P_{121}^{*}(x, \xi) \psi_{1}(\xi)+P_{123}^{*}(x, \xi) \psi_{3}(\xi)\right] d \xi=-\frac{\bar{T}(x)}{D}, \\
& f_{-a}^{a}\left[P_{321}^{*}(x, \xi) \psi_{1}(\xi)+P_{323}^{*}(x, \xi) \psi_{3}(\xi)\right] d \xi=-\frac{\bar{Q}(x)}{D},
\end{aligned}
$$

where $\bar{M}(x), \bar{T}(x)$ and $\bar{Q}(x)$ are applied bending, twisting moments and shear force on the crack surface, respectively. Because of the singularity $O\left(r^{-1 / 2}\right)$ of the stress resultants at the crack tips $x= \pm a$ [40], the displacement discontinuity of the crack can be approximated as

$$
\psi_{k}(\xi)=\sqrt{a^{2}-\xi^{2}} \sum_{l=0}^{L} c_{k, l} U_{l}(\xi / a), \quad-a \leq \xi \leq a,
$$

where $c_{k, l}$ represents the coefficient for different fracture modes, $U_{k}(x / a)$ are the Chebyshev polynomials of the second kind as

$$
U_{l}(\xi / a)=\frac{\sin [(l+1) \arccos (\xi / a)]}{\sin [\arccos (\xi / a)]} .
$$

Considering the integral formula given by Kaya and Erdogan ${ }^{28}$, we have 


$$
f_{-a}^{a} \frac{\sqrt{a^{2}-\xi^{2}} U_{l}(\xi / a)}{(\xi-x)^{2}} d \xi=-\pi(l+1) U_{l}(x / a)
$$

and

$$
\int_{-a}^{a} \frac{\sqrt{a^{2}-\xi^{2}} U_{l}(\xi / a)}{\xi-x} d \xi=-\pi T_{l+1}(x / a)
$$

where $T_{n}(x)$ are the Chebyshev polynomials of the first kind. Taking the integral equation (23) at collocation points

$$
x^{(m)} / a=\cos \frac{(2 m+1)}{2(L+1)} \pi, \quad m=0,1,2, \ldots, L,
$$

we obtain

$$
\sum_{l=0}^{L} c_{2, l}\left[-\pi(l+1) U_{l}\left(x^{(m)} / a\right) g_{222}+\int_{-a}^{a} E_{2}\left(x^{(m)}, \xi\right) \sqrt{a^{2}-\xi^{2}} U_{l}(\xi / a) d \xi\right]=-\frac{\bar{M}\left(x^{(m)}\right)}{D}, m=0,1, \ldots, L,
$$

where

$$
E_{2}\left(x^{(m)}, \xi\right)=P_{222}^{*}\left(x^{(m)}, \xi\right)-\frac{g_{222}}{\left(\xi-x^{(m)}\right)^{2}} .
$$

It is clear that there is a weak singularity $O(\ln r)$ in $E_{2}(x, \xi)$, thus a coordinate transformation is required to cancel the weak singularity with $\xi^{\prime}=\sqrt{\xi-x}$. Therefore, Eq.(30) provides a set of linear algebraic equations to determine $L+1$ unknown coefficients $c_{2, l}$. From the definition of stress intensity factor at the crack tip, we have

$$
K_{\mathrm{I}}^{b}( \pm a)=\frac{E( \pm a) h^{3} \sqrt{\pi}}{48 \sqrt{2}} \lim _{r \rightarrow 0} \frac{\psi_{2}( \pm a)}{\sqrt{r}},
$$

in which $E( \pm a)$ indicates Young's modulus at the crack tips. Considering Eq.(25), we can obtain the stress intensity factors directly with the slope discontinuity $\psi_{2}$ by

$$
K_{\mathrm{I}}^{b}( \pm a)=\frac{E( \pm a) h^{3}}{48} \sum_{l=0}^{L} c_{2, l} U_{l}( \pm 1) \sqrt{\pi a}
$$

where $U_{l}(+1)=l+1$ and $U_{l}(-1)=(-1)^{l}(l+1)$. Considering the twist moment and shear force in Eq.(24), the integral kernels of hyper and strong singularities from Eq.(24) are obtained 


$$
P_{i 2 k}^{*}(x, \xi)=\frac{g_{i 2 k}(x, \xi)}{(\xi-x)^{2}}+\frac{f_{i 2 k}(x, \xi)}{\xi-x}+E_{i k}(x, \xi),
$$

where $E_{i k}(x, \xi)$ is of weak singularity of $O(\ln r)$. Therefore, the coupled integral equations are written as

$$
\begin{aligned}
& \sum_{l=0}^{L} c_{1, l}\left[-\pi(l+1) U_{l}\left(x^{(m)} / a\right) g_{121}-\pi T_{l+1}\left(x^{(m)} / a\right) f_{121}+\int_{-a}^{a} E_{11}\left(x^{(m)}, \xi\right) \sqrt{a^{2}-\xi^{2}} U_{l}(\xi / a) d \xi\right] \\
& +\sum_{l=0}^{L} c_{3, l}\left[-\pi(l+1) U_{l}\left(x^{(m)} / a\right) g_{123}-\pi T_{l+1}\left(x^{(m)} / a\right) f_{123}+\int_{-a}^{a} E_{13}\left(x^{(m)}, \xi\right) \sqrt{a^{2}-\xi^{2}} U_{l}(\xi / a) d \xi\right] \\
& =-\frac{\bar{T}\left(x^{(m)}\right)}{D}, \quad m=0,1, \ldots, L . \\
& \sum_{l=0}^{L} c_{1, l}\left[-\pi(l+1) U_{l}\left(x^{(m)} / a\right) g_{321}-\pi T_{l+1}\left(x^{(m)} / a\right) f_{321}+\int_{-a}^{a} E_{31}\left(x^{(m)}, \xi\right) \sqrt{a^{2}-\xi^{2}} U_{l}(\xi / a) d \xi\right] \\
& +\sum_{l=0}^{L} c_{3, l}\left[-\pi(l+1) U_{l}\left(x^{(m)} / a\right) g_{323}-\pi T_{l+1}\left(x^{(m)} / a\right) f_{323}+\int_{-a}^{a} E_{33}\left(x^{(m)}, \xi\right) \sqrt{a^{2}-\xi^{2}} U_{l}(\xi / a) d \xi\right] \\
& =-\frac{\bar{Q}\left(x^{(m)}\right)}{D}, \quad \\
& m=0,1, \ldots, L .
\end{aligned}
$$

Similarly to mode I, the mixed mode fractures under twisting moment and shear force can be solved numerically. The mixed mode stress intensity factors are obtained by

$$
K_{\mathrm{II}}^{b}( \pm a)=\frac{E( \pm a) h^{3}}{48} \sum_{l=0}^{L} c_{1, l} U_{l}( \pm 1) \sqrt{\pi a}
$$

and

$$
K_{\mathrm{III}}^{b}( \pm a)=\frac{5 E( \pm a) h}{24(1+v)} \sum_{l=0}^{L} c_{3, l} U_{l}( \pm 1) \sqrt{\pi a} .
$$

It is worth noting that the singular stresses vary along the thickness of the plate (coordinate $\left.x_{3}\right)$. The stress intensity factors above can be related to the stress intensity factors through the following relationships:

$$
K_{\mathrm{I}}=\frac{12 x_{3}}{h^{3}} K_{\mathrm{I}}^{b}, \quad K_{\mathrm{II}}=\frac{12 x_{3}}{h^{3}} K_{\mathrm{II}}^{b}, \quad K_{\mathrm{III}}=\frac{3}{2 h}\left[1-\left(\frac{2 x_{3}}{h}\right)^{2}\right] K_{\mathrm{III}}^{b} .
$$




\section{Example 1. Embedded crack under various loads}

We assume that a crack embedded in an infinite homogenous plate is subjected to uniform bending moment $M_{0}$, twist moment $T_{0}$ and shear force $Q_{0}$, respectively. Firstly, the uniform bending moment $M_{0}$ is considered and the numerical results of normalized stress intensity factor $K_{\mathrm{I}}^{(b)} / M_{0} \sqrt{\pi a}$ is shown in Figure 2 versus the normalized plate thickness $\lambda_{0}(=h / \sqrt{10} a)$. The maximum number of the Chebyshev polynomials is only one free parameter $L$ in the numerical computational process. The convergence of the numerical result is shown in Table 1. The solution from the handbook [41] gives $K_{0}^{b} / M_{0} \sqrt{\pi a}=0.79$. The relative error is less than $0.5 \%$ when the maximum number $L \geq 3$. For the constant twisting moment $T_{0}$ acting on the crack surface, the normalized stress intensity factors $K_{\mathrm{II}}^{b} / T_{0} \sqrt{\pi a}$ and $K_{\mathrm{III}}^{b}(1+v) \lambda_{0} a$ $/ T_{0} \sqrt{\pi a}$ versus the parameter $\lambda_{0}$ are shown in Figure 3(a) and (b) respectively, with the maximum number of the Chebyshev polynomials $L=10$. Finally, consider a constant shear force $Q_{0}$ on the crack surfaces, the normalized stress intensity factors $K_{\mathrm{II}}^{b} / Q_{0} a \sqrt{\pi a}$ and $K_{\mathrm{III}}^{b}$ $(1+v) \lambda_{0} / Q_{0} \sqrt{\pi a}$ are presented in Figure 4(a) and (b) respectively.

Table 1. Convergence observation when $\lambda_{0}=0.5, v=0.25$.

\begin{tabular}{cccccc}
\hline$L$ & 0 & 1 & 2 & 3 & 4 \\
\hline$K_{\mathrm{I}}^{b} / M_{0} \sqrt{\pi a}$ & 0.6723 & 0.7746 & 0.7931 & 0.7940 & 0.7940 \\
\hline
\end{tabular}




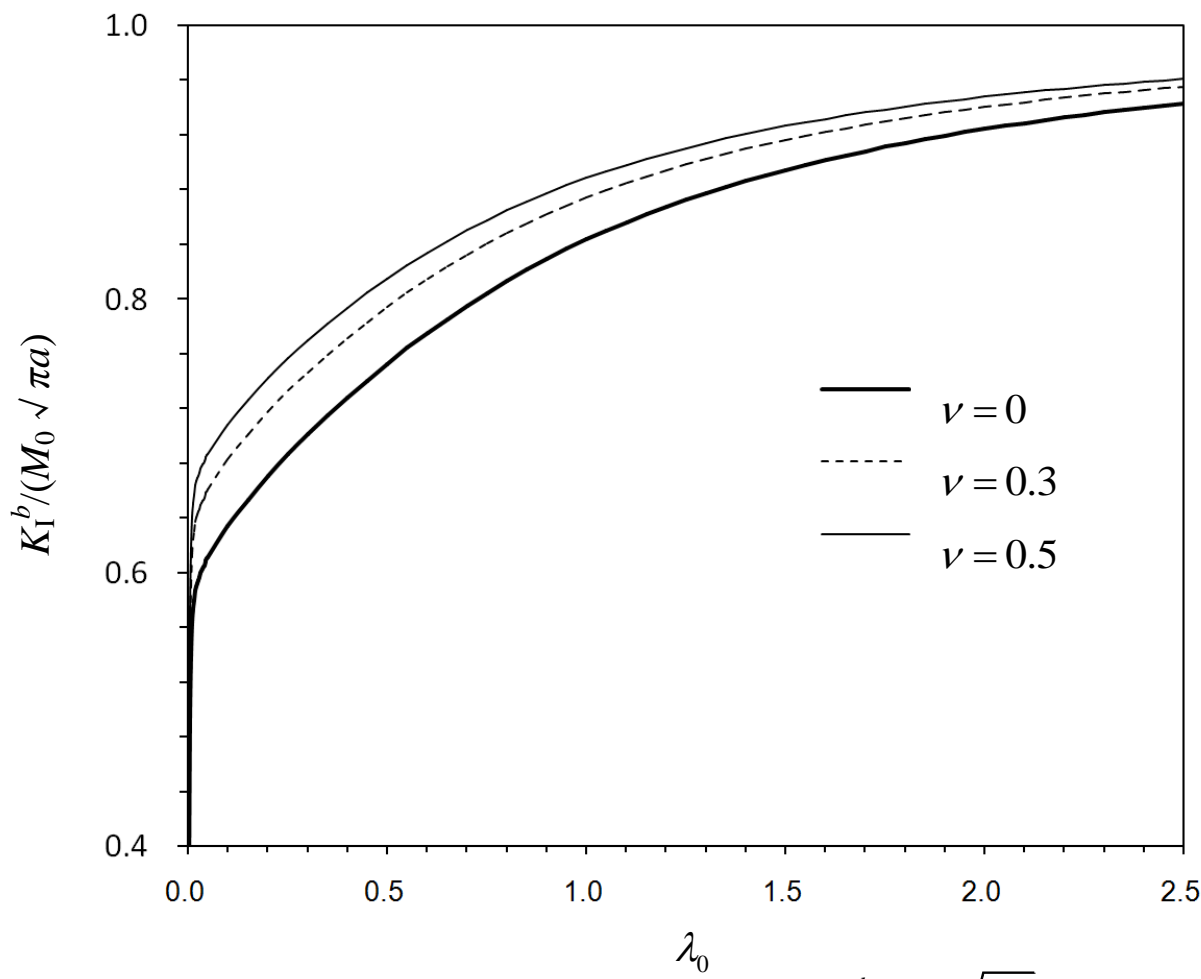

Figure 2. Normalized stress resultant intensity factor $K_{\mathrm{I}}^{b} / M_{0} \sqrt{\pi a}$ under a bending moment $M_{0}$.

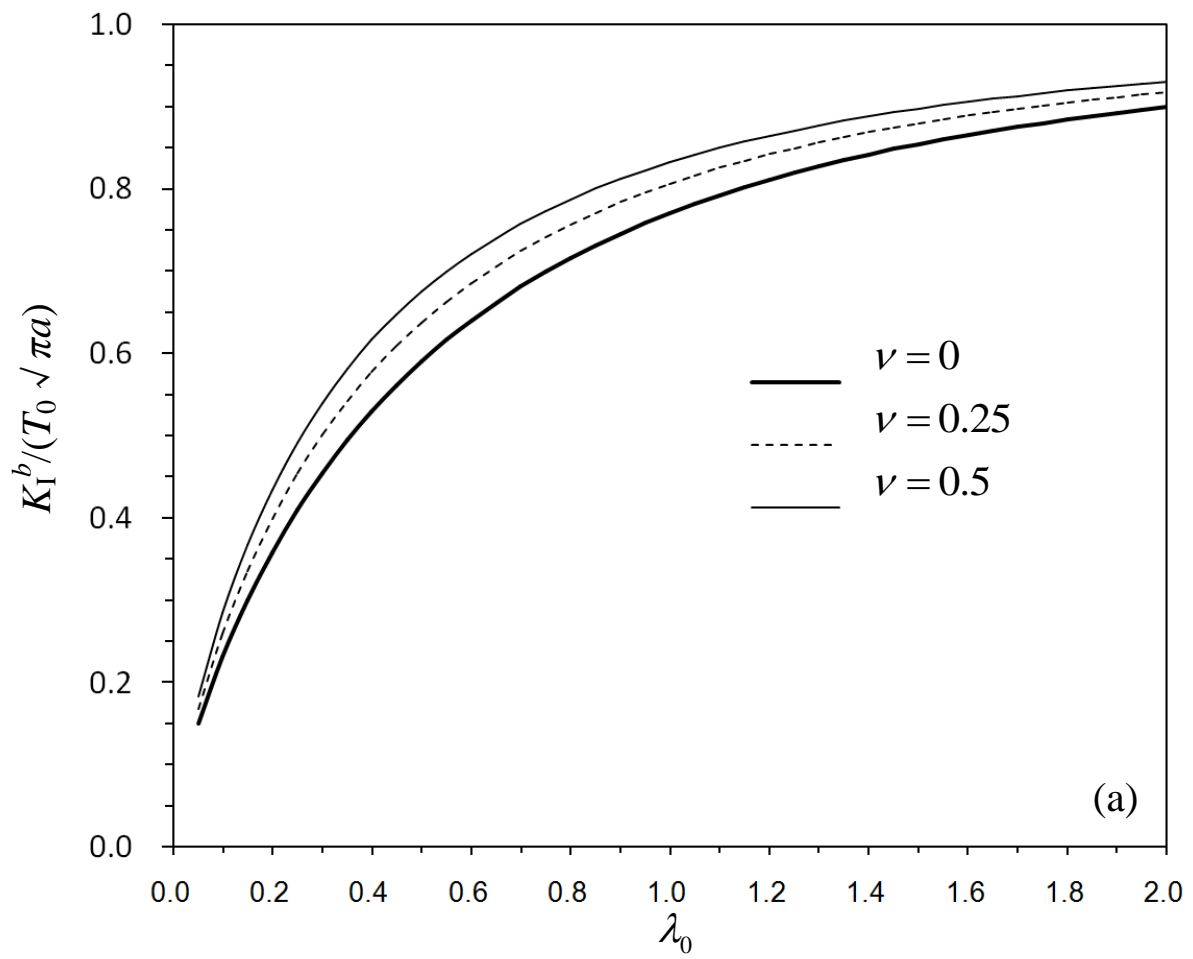




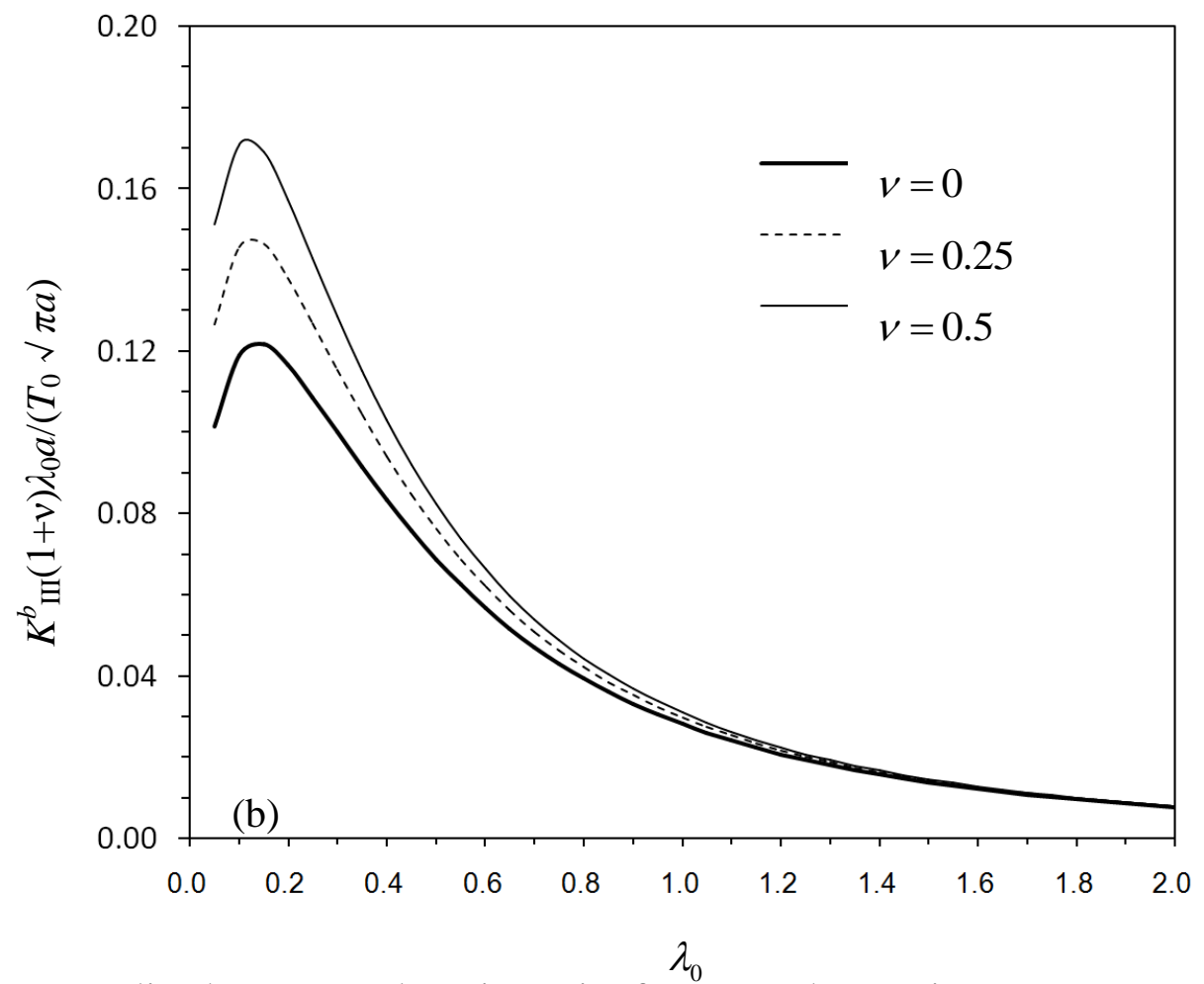

Figure 3. Normalized stress resultant intensity factors under a twist moment $T_{0}$ : (a) $K_{\mathrm{II}}^{b} / T_{0} \sqrt{\pi a}$; (b) $K_{\mathrm{III}}^{b}(1+v) \lambda_{0} a / T_{0} \sqrt{\pi a}$.

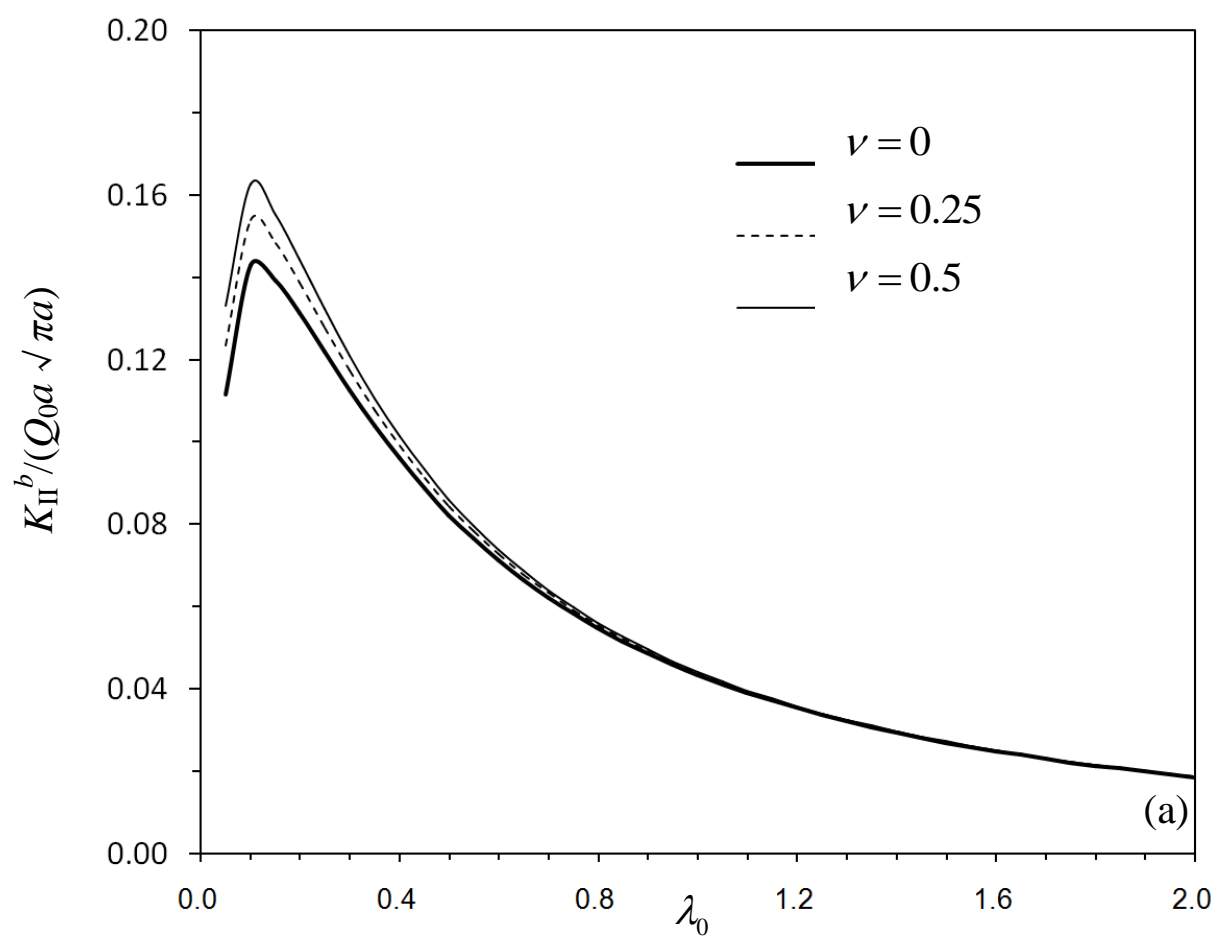




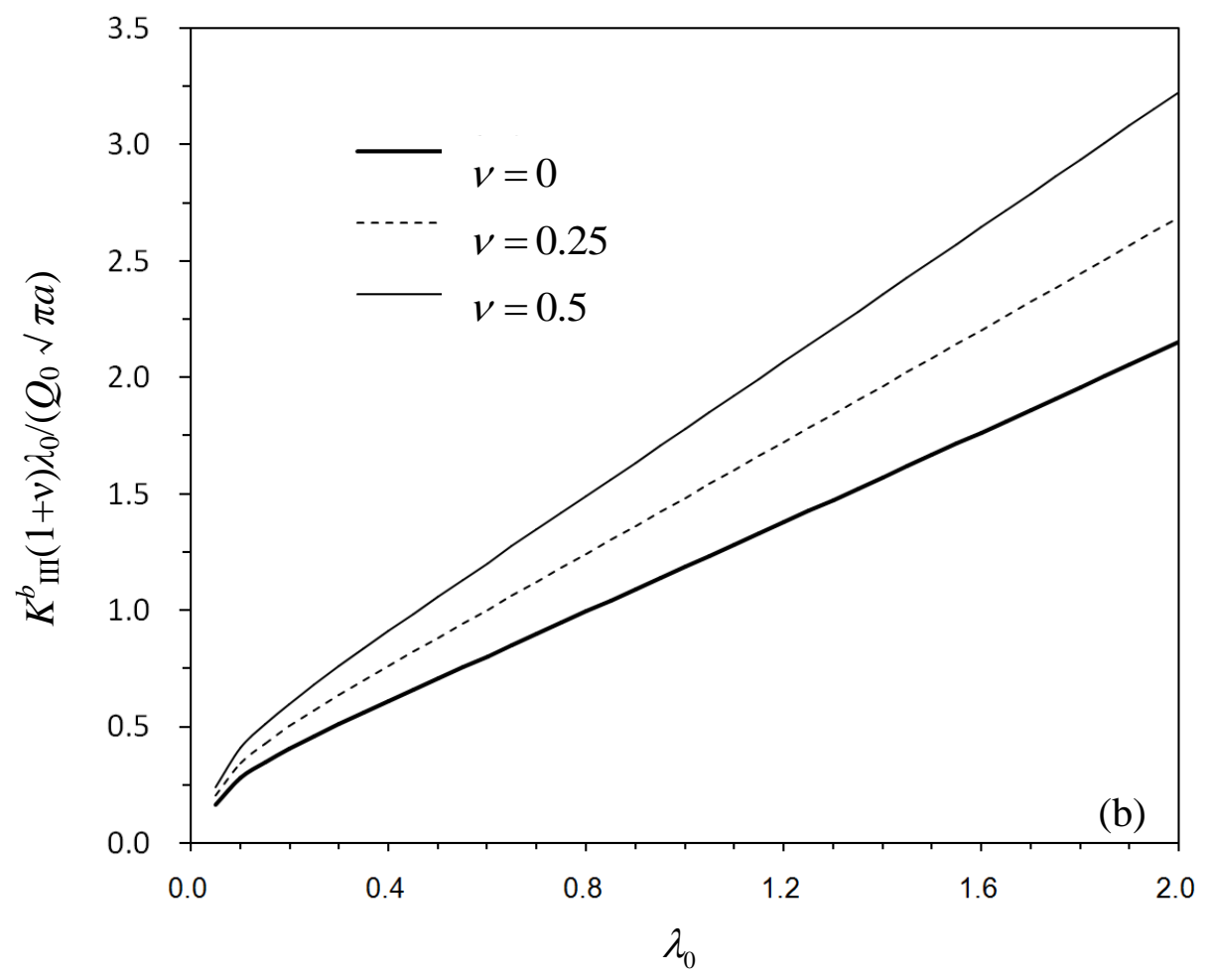

Figure 4. Normalized stress resultant intensity factor under a shear force $Q_{0}$ : (a) $K_{\mathrm{II}}^{b} / Q_{0} a \sqrt{\pi a} ;(\mathrm{b}) K_{\mathrm{III}}^{b}(1+v) \lambda_{0} / Q_{0} \sqrt{\pi a}$.

\section{Hybrid method for crack in FGM plate}

Simple examples in chapter 3 have shown the accuracy obtained for the stress intensity factors by DDM with the Chebyshev polynomials in homogeneous body. However, that approach is not applicable to the continuously non-homogenous media due to the absence of explicit expressions of fundamental solutions (integral kernels). It is known that the crack tip behaviour in continuously non-homogeneous media has the same characteristics as in homogeneous ones with the only need to take into account the material coefficients at the crack tip [42]. In order to utilize the DDM formulation with the Chebyshev polynomials, also in the case of FGM, the general solutions of the displacements in non-homogeneous media are decomposed into two parts, as follows

$$
w_{i}=w_{i}^{*}+w_{i}^{m}
$$


where the superscripts "*" and " $m$ " represent the solutions given by the DDM (described in chapter 3) and the meshless methods, respectively. Then, the bending and twisting moments, and shear forces become

$$
M_{\alpha \beta}=M_{\alpha \beta}^{*}+M_{\alpha \beta}^{m}, \quad Q_{\alpha}=Q_{\alpha}^{*}+Q_{\alpha}^{m} .
$$

in which $M_{\alpha \beta}^{*}, Q_{\alpha}^{*}$ and $M_{\alpha \beta}^{m}, Q_{\alpha}^{m}$ are defined by (1) with replacing $w_{i}$ by $w_{i}^{*}$ and $w_{i}^{m}$, respectively, and taking $D(\mathbf{x})=E(\mathbf{x}) h^{3} / 12\left(1-v^{2}\right), C(\mathbf{x})=D(\mathbf{x})(1-v) \lambda^{2} / 2$. Then, according to Eq. (5), the solutions for homogeneous plate $M_{\alpha \beta}^{*}, Q_{\alpha}^{*}$ satisfy the equations

$$
D\left(\frac{M_{\alpha \beta}^{*}}{D}\right)_{, \beta}-Q_{\alpha}^{*}=0 . C\left(\frac{Q_{\alpha}^{*}}{C}\right)_{, \alpha}+q^{*}=0
$$

Furthermore, the governing Eqs. (3) yield

$$
D_{, \beta} \frac{M_{\alpha \beta}}{D}+D\left(\frac{M_{\alpha \beta}}{D}\right)_{, \beta}-Q_{\alpha}=0, C_{, \alpha} \frac{Q_{\alpha}}{C}+C\left(\frac{Q_{\alpha}}{C}\right)_{, \alpha}+q=0 .
$$

Hence and from (41)-(42), assuming $q^{*}=0$, one obtains

$$
D_{, \beta} \frac{M_{\alpha \beta}}{D}+D\left(\frac{M_{\alpha \beta}^{m}}{D}\right)_{, \beta}-Q_{\alpha}^{m}=0, \quad C_{, \alpha} \frac{Q_{\alpha}}{C}+C\left(\frac{Q_{\alpha}^{m}}{C}\right)_{, \alpha}+q=0
$$

or in view of (41) and the definitions of $M_{\alpha \beta}^{*}, Q_{\alpha}^{*}$ and $M_{\alpha \beta}^{m}, Q_{\alpha}^{m}$, these equations become

$$
\begin{aligned}
& \frac{(1-v) D w_{\alpha, \beta \beta}^{m}}{2}+\frac{(1+v) D w_{\beta, \alpha \beta}^{m}}{2}-C\left(w_{\alpha}^{m}+w_{3, \alpha}^{m}\right)+\frac{D_{, \beta}}{D}\left(M_{\alpha \beta}^{*}+M_{\alpha \beta}^{m}\right)=0 \\
& C\left(w_{\alpha, \alpha}^{m}+w_{3, \beta \beta}^{m}\right)+\frac{D_{, \alpha}}{D}\left(Q_{\alpha}^{*}+Q_{\alpha}^{m}\right)+q=0
\end{aligned}
$$

where we have utilized the relationship $C_{, \alpha}(\mathbf{x}) / C(\mathbf{x})=D_{, \alpha}(\mathbf{x}) / D(\mathbf{x})$. Applying the governing equations (43) to the problem with a straight crack in infinite medium and making use of the integral representation of the bending stress-couples $M_{\alpha \beta}^{*}(\mathbf{x})$ and shear stress-resultants $Q_{\alpha}^{*}(\mathbf{x})$ according to Eqs (15) and (16), using the Chebyshev polynomials for approximation of displacement discontinuities $\psi_{k}(\xi)$ by (25), one can re-cast the governing equations (43) into the form 


$$
\begin{array}{r}
\frac{D(\mathbf{x})}{2}\left[(1-v) w_{\alpha, \beta \beta}^{m}(\mathbf{x})+(1+v) w_{\beta, \alpha \beta}^{m}(\mathbf{x})\right]-C(\mathbf{x})\left(w_{\alpha}^{m}(\mathbf{x})+w_{3, \alpha}^{m}(\mathbf{x})\right)+\frac{D_{, \beta}(\mathbf{x})}{D(\mathbf{x})} M_{\alpha \beta}^{m}(\mathbf{x})- \\
-D_{, \beta}(\mathbf{x}) \sum_{l=0}^{L} \int_{-a}^{a} P_{\alpha \beta k}^{*}(\mathbf{x}, \xi) c_{k, l} \sqrt{a^{2}-\xi^{2}} U_{l}(\xi) d \xi=0 \\
C(\mathbf{x})\left(w_{\alpha, \alpha}^{m}+w_{3, \beta \beta}^{m}\right)+\frac{D_{, \alpha}(\mathbf{x})}{D(\mathbf{x})} Q_{\alpha}^{m}(\mathbf{x})+D_{, \alpha}(\mathbf{x}) \sum_{l=0}^{L} \int_{-a}^{a} P_{3 \alpha k}^{*}(\mathbf{x}, \xi) c_{k, l} \sqrt{a^{2}-\xi^{2}} U_{l}(\xi) d \xi+q=0
\end{array}
$$

Two kinds of boundary conditions are considered as follows

(1) Displacement boundary condition on $\Gamma_{w}$ :

$$
w_{k}^{*}+w_{k}^{m}=\bar{w}_{k}(\mathbf{x}),(k=1,2,3), \quad \mathbf{x} \in \Gamma_{w},
$$

where $\bar{w}_{k}$ indicate the slopes and deflection specified on the displacement boundary $\Gamma_{w}$.

(2) Traction boundary condition on $\Gamma_{p}$ :

$$
\left(M_{\alpha \beta}^{*}+M_{\alpha \beta}^{m}\right) n_{\beta}=\bar{p}_{\alpha}(\mathbf{x}) \quad \text { and }\left(Q_{\alpha}^{*}+Q_{\alpha}^{m}\right) n_{\alpha}=\bar{p}_{3}(\mathbf{x}), \quad \mathbf{x} \in \Gamma_{p},
$$

where $\bar{p}_{k}$ are the moments and shear force specified on the traction boundary $\Gamma_{p}$. The integrals in Eq. (44) are regular as the integral kernels are regular as long as $\mathbf{x} \notin \Gamma_{c}$. However, when the source point is located on the crack surface, i.e. $\mathbf{x} \in \Gamma_{c}$, the traction boundary conditions become

$$
\begin{aligned}
& \sum_{l=0}^{L} c_{2, l}\left[-\pi(l+1) U_{l}(x / a) g_{222}+\int_{-a}^{a} E_{2}(x, \xi) \sqrt{a^{2}-\xi^{2}} U_{l}(\xi / a) d \xi\right]+\frac{M_{22}^{m}(x)}{D}=-\frac{\bar{M}(x)}{D} \\
& \sum_{l=0}^{L} c_{1, l}\left[-\pi(l+1) U_{l}\left(x_{m} / a\right) g_{121}-\pi T_{l+1}\left(x_{m} / a\right) f_{121}+\int_{-a}^{a} E_{11}\left(x_{m}, \xi\right) \sqrt{a^{2}-\xi^{2}} U_{l}(\xi / a) d \xi\right] \\
& +\sum_{l=0}^{L} c_{3, l}\left[-\pi(l+1) U_{l}(x / a) g_{123}-\pi T_{l+1}(x / a) f_{123}+\int_{-a}^{a} E_{13}(x, \xi) \sqrt{a^{2}-\xi^{2}} U_{l}(\xi / a) d \xi\right] \\
& +\frac{M_{12}^{m}(x)}{D}=-\frac{\bar{T}(x)}{D}, \\
& \sum_{l=0}^{L} c_{1, l}\left[-\pi(l+1) U_{l}\left(x_{m} / a\right) g_{321}-\pi T_{l+1}\left(x_{m} / a\right) f_{321}+\int_{-a}^{a} E_{31}\left(x_{m}, \xi\right) \sqrt{a^{2}-\xi^{2}} U_{l}(\xi / a) d \xi\right]
\end{aligned}
$$




$$
\begin{aligned}
& +\sum_{l=0}^{L} c_{3, l}\left[-\pi(l+1) U_{l}(x / a) g_{323}-\pi T_{l+1}(x / a) f_{323}+\int_{-a}^{a} E_{33}(x, \xi) \sqrt{a^{2}-\xi^{2}} U_{l}(\xi / a) d \xi\right] \\
& +\frac{Q_{2}^{m}(x)}{D}=-\frac{\bar{Q}(x)}{D} .
\end{aligned}
$$

Thus, the task is to solve the boundary value problem for the partial differential equations with variable coefficients, in order to get "meshless" displacements.

\section{Meshless approaches for FGMs}

Two meshless approaches will be presented: (i) the Point Collocation Method as a strong meshless formulation of the boundary value problem; (ii) MLPG (Meshless Local PetrovGalerkin method) as a local weak formulation. In both approaches, we employ the polynomial interpolation of "meshless" field variables within a finite block, using the standard Lagrange finite elements [43-45].

\subsection{Hybrid MDDM with Point Collocation Method (PCM)}

Considering a block shown in Figure 5, we have a quadratic block with area $\Omega$ in physical domain mapped into a square in the intrinsic space, by using quadratic shape functions with 8 seeds. Then the coordinate transform (mapping) can be written as

$$
x_{\alpha}=\sum_{k=1}^{8} N_{k}\left(\eta_{1}, \eta_{2}\right) x_{\alpha}^{(k)}
$$

where $N_{k}\left(\eta_{1}, \eta_{2}\right)$ is the shape function, $\left(x_{1}^{(k)}, x_{2}^{(k)}\right)$ are the coordinates of the seed $k$. For the first order partial differentials of function $f\left(x_{1}, x_{2}\right)$, one has

$$
\frac{\partial f}{\partial x_{1}}=\frac{1}{J}\left(\beta_{11} \frac{\partial f}{\partial \eta_{1}}+\beta_{12} \frac{\partial f}{\partial \eta_{2}}\right), \quad \frac{\partial f}{\partial x_{2}}=\frac{1}{J}\left(\beta_{21} \frac{\partial f}{\partial \eta_{1}}+\beta_{22} \frac{\partial f}{\partial \eta_{2}}\right),
$$

where

$$
\beta_{11}=\frac{\partial x_{2}}{\partial \eta_{2}}, \beta_{12}=-\frac{\partial x_{2}}{\partial \eta_{1}}, \beta_{21}=-\frac{\partial x_{1}}{\partial \eta_{2}}, \beta_{22}=\frac{\partial x_{1}}{\partial \eta_{1}}, J=\beta_{22} \beta_{11}-\beta_{21} \beta_{12} .
$$

Consider a set of two dimensional uniformly distributed nodes shown in Figure 5(a), $\eta_{1}^{(i)}=-1+2(i-1) /\left(L_{1}-1\right), i=1,2, \ldots, L_{1}$ and $\eta_{2}^{(j)}=-1+2(j-1) /\left(L_{2}-1\right), j=1,2, \ldots, L_{2}$, in which $L_{1}$ and $L_{2}$ are the number of nodes distributed along $\eta_{1}$ and $\eta_{2}$ axes respectively. The number 
of nodes in total is $L_{m}=L_{1} \times L_{2}$. To interpolate any continuous function $f\left(x_{1}, x_{2}\right)$ with Lagrange series, one has

$$
f\left(x_{1}, x_{2}\right)=\sum_{i=1}^{L_{1}} \sum_{j=1}^{L_{2}} \prod_{\substack{m=1 \\ m \neq i}}^{L_{1}} \frac{\left(\eta_{1}-\eta_{1}^{(m)}\right)}{\left(\eta_{1}^{(i)}-\eta_{1}^{(m)}\right)} \prod_{\substack{n=1 \\ n \neq j}}^{L_{2}} \frac{\left(\eta_{2}-\eta_{2}^{(n)}\right)}{\left(\eta_{2}^{(j)}-\eta_{2}^{(n)}\right)} f^{(i j)},
$$

where the superscript $(i j)$ indicates the global number of node $i j=(j-1) L_{1}+i$. Then, the first order partial differential is determined easily by

$$
\frac{\partial f}{\partial x_{\alpha}}=\frac{1}{J} \sum_{i=1}^{L_{1}} \sum_{j=1}^{L_{2}}\left[\beta_{\alpha 1} \frac{\partial F_{i}\left(\eta_{1}\right)}{\partial \eta_{1}} G_{j}\left(\eta_{2}\right)+\beta_{\alpha 2} F_{i}\left(\eta_{1}\right) \frac{\partial G_{j}\left(\eta_{2}\right)}{\partial \eta_{2}}\right] f^{(i j)}
$$

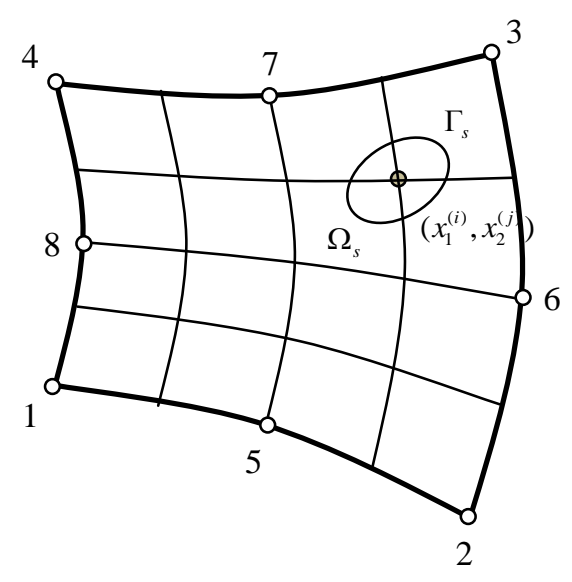

(a)

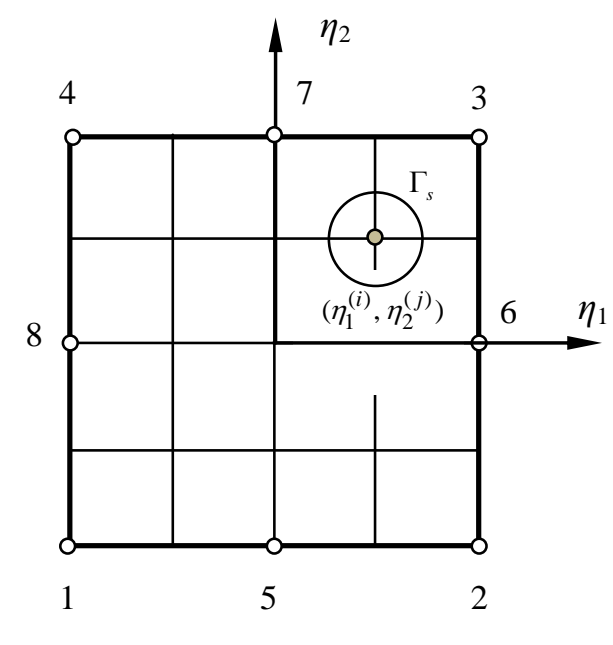

(b)

Figure 5. Mapping and node distribution: (a) physical quadratic block; (b) square domain in intrinsic space with 8 seeds. ○ mapping seeds; o node.

where $\beta_{\alpha \beta}$ and $J$ are given in Eq.(52). Changing $f^{(i j)}$ with $\left(\partial f / \partial x_{\alpha}\right)^{(i j)}$ in Eq.(54), any higher order partial differentials at collocation points can be obtained in terms of the nodal values of function $f$. Substituting those first order and second order partial derivatives into the governing equations in Eqs $(44,47,48,49)$ and boundary conditions, we obtain the linear algebraic equations with unknown nodal slopes $w_{\alpha}^{(i j)}$, deflection $w_{3}^{(i j)}$, and coefficients $c_{k, l}$. By solving those linear algebraic equations, we receive all unknown coefficients and displacements in the analyzed domain, which can be used to determine mixed modes stress intensity factors from Eqs (33), (37) and (38) respectively. 


\subsection{Hybrid MDDM with $M L P G$}

Consider a flat FGM plate with the neutral plane of the plate $x_{3}=0$, on which the normal bending stress is zero and the governing equations (3) can be written in weak form as

$$
\int_{\Omega_{s}^{i}}\left(M_{\alpha \beta, \beta}-Q_{\alpha}\right) \hat{u} d \Omega=0, \quad \int_{\Omega_{s}^{i}}\left(Q_{\alpha, \alpha}+q\right) \hat{u} d \Omega=0,
$$

where $\hat{u}$ is a test function, which is different from zero in the local integral domain. By the divergence theorem, Eq. (55) can be rewritten as

$$
\int_{\Gamma_{s}^{i}} M_{\alpha \beta} n_{\beta} \widehat{u} d \Gamma-\int_{\Omega_{s}^{i}}\left[M_{\alpha \beta} \hat{u}_{, \alpha}+Q_{\alpha} \hat{u}\right] d \Omega=0, \int_{\Gamma_{s}^{i}} Q_{\alpha} n_{\alpha} \widehat{u} d \Gamma-\int_{\Omega_{s}^{i}}\left[Q_{\alpha} \hat{u}_{, \alpha}+q \hat{u}\right] d \Omega=0
$$

where $n_{\alpha}$ is the component of normal outward to the boundary $\Gamma_{s}^{i}$ of the local integral domain $\Omega_{s}^{i}$. The simplest choice of the test function is selected as a unit step function in the local integral domain as follows

$$
\widehat{u}(\mathbf{x})= \begin{cases}1 & \text { at } \mathbf{x} \in \Omega_{s}^{i}, \\ 0 & \text { at } \mathbf{x} \notin \Omega_{s}^{i} .\end{cases}
$$

Then, the governing equations in Eq. (56) become

$$
\int_{\Gamma_{s}^{i}} M_{\alpha \beta} n_{\beta} d \Gamma-\int_{\Omega_{s}^{i}} Q_{\alpha} d \Omega=0, \quad \int_{\Gamma_{s}^{i}} Q_{\alpha} n_{\alpha} d \Gamma-\int_{\Omega_{s}^{i}} q d \Omega=0 .
$$

Considering the identity

$$
\left(x_{\alpha} Q_{\beta}\right)_{, \beta}=Q_{\alpha}+x_{\alpha} Q_{\beta, \beta}
$$

and governing equation (3), we have

$$
Q_{\alpha}=\left(x_{\alpha} Q_{\beta}\right)_{, \beta}+x_{\alpha} q \text {. }
$$

Then, we obtained the domain integral

$$
\int_{\Omega_{s}^{i}} Q_{\alpha} d \Omega=\int_{\Gamma_{s}^{i}} x_{\alpha} Q_{\beta} n_{\beta} d \Gamma+\int_{\Omega_{s}^{i}} x_{\alpha} q d \Omega .
$$

Therefore, the integration in Eq. (58) can be simplified as

$$
\int_{\Gamma_{s}^{i}}\left(M_{\alpha \beta}-x_{\alpha} Q_{\beta}\right) n_{\beta} d \Gamma-\int_{\Omega_{s}^{i}} q x_{\alpha} d \Omega=0 .
$$

If the pressure load is constant, i.e. $q=q_{0}$, we have 


$$
\begin{aligned}
& \int_{\Gamma_{s}^{i}}\left(M_{\alpha \beta}-x_{\alpha} Q_{\beta}\right) n_{\beta} d \Gamma=q_{0} \bar{x}_{\alpha}^{(i)} \Omega_{s}^{i}, \\
& \int_{\Gamma_{s}^{i}} Q_{\alpha} n_{\alpha} d \Gamma=q_{0} \Omega_{s}^{i},
\end{aligned}
$$

in which $\left(\bar{x}_{1}^{(i)}, \bar{x}_{2}^{(i)}\right)$ indicates the centroid of the local integral domain $\Omega_{s}^{i}$. It is worth to point out that the stress resultants $M_{\alpha \beta}$ and $Q_{\alpha}$ are given by Eq. (41) with $M_{\alpha \beta}^{*}, Q_{\alpha}^{*}$ being known from the DDM formulation with the Chebyshev polynomials, while

$$
\begin{aligned}
& M_{\alpha \beta}^{m}=\frac{(1-v) D}{2}\left(w_{\alpha, \beta}^{m}+w_{\beta, \alpha}^{m}+\frac{2 v}{1-v} w_{\gamma, \gamma}^{m} \delta_{\alpha \beta}\right), \\
& Q_{\alpha}^{m}=C\left(w_{\alpha}^{m}+w_{3, \alpha}^{m}\right),
\end{aligned}
$$

where the bending stiffness $D=D(\mathbf{x})$ for non-homogenous media and displacements $\left(w_{1}^{m}, w_{2}^{m}, w_{3}^{m}\right)$ are approximated by the Lagrange series interpolation from Eq.(53) in terms of the nodal values.

For the sake of simplicity, the local integral domain is selected as a circle of radius $r_{0}$ centered at $\left(\eta_{1}^{(i)}, \eta_{2}^{(j)}\right)$ which corresponds to the point $\mathbf{x}^{(i j)}$ in the physical domain, where $r_{0}$ is free parameter in MLPG. A point on the local integral boundary $S^{\prime}\left(\eta_{1}^{s}, \eta_{2}^{s}\right)$ shown in Figure 6, is

$$
\eta_{\alpha}^{s}=\eta_{\alpha}^{(i)}+r_{0} n_{\alpha}, n_{\alpha}=\delta_{\alpha 1} \cos \theta+\delta_{\alpha 2} \sin \theta
$$

and its coordinates in the physical domain are

$$
x_{\alpha}^{s}=\sum_{k=1}^{8} N_{k}\left(\eta_{1}^{s}, \eta_{2}^{s}\right) x_{\alpha}^{(k)} .
$$

Apparently, the different segments of global coordinates

$$
\begin{aligned}
& d x_{1}^{s}=r_{0} d \theta \sum_{k=1}^{8}\left(-\frac{\partial N_{l}}{\partial \eta_{1}} \sin \theta+\frac{\partial N_{l}}{\partial \eta_{2}} \cos \theta\right) x_{1}^{(k)}=g_{1}\left(\eta_{1}^{s}, \eta_{2}^{s}\right) r_{0} d \theta \\
& d x_{2}^{s}=r_{0} d \theta \sum_{k=1}^{8}\left(-\frac{\partial N_{l}}{\partial \eta_{1}} \sin \theta+\frac{\partial N_{l}(\xi, \eta)}{\partial \eta} \cos \theta\right) x_{2}^{(k)}=g_{2}\left(\eta_{1}^{s}, \eta_{2}^{s}\right) r_{0} d \theta
\end{aligned}
$$

correspond to differentials $d \eta_{\alpha}^{s}=\tau_{\alpha} r_{0} d \theta$ along the circle $\Gamma_{s}^{\prime i}$ with tangent vector $\tau_{\alpha}=-\delta_{\alpha 1} \sin \theta+\delta_{\alpha 2} \cos \theta$. Thus, the integration segment is 


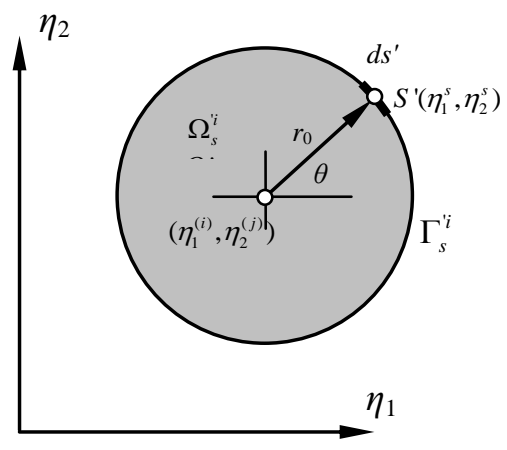

(a)

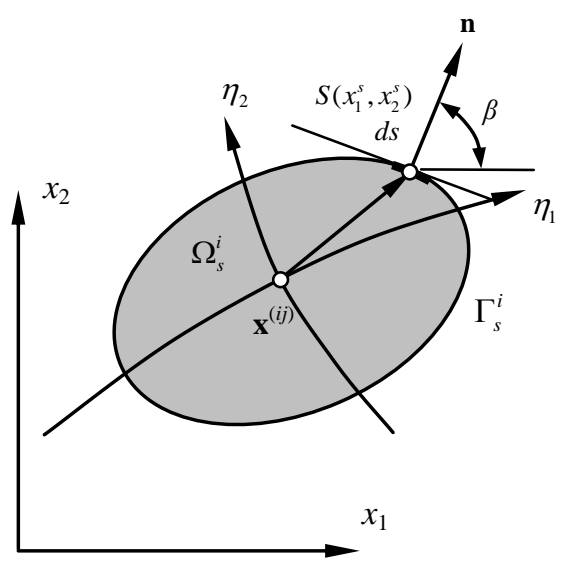

(b)

Figure 6. Local integral domain and its boundary: (a) circle in normalized coordinate; (b) physical local integral domain.

$$
d s=\sqrt{\left(d x_{1}^{s}\right)^{2}+\left(d x_{2}^{s}\right)^{2}}=\sqrt{\left(g_{1}\right)^{2}+\left(g_{2}\right)^{2}} r_{0} d \theta=J^{\prime} d \theta
$$

and the tangential at point $S\left(x_{1}^{s}, x_{2}^{s}\right)$ on the boundary

$$
t_{\alpha}=d x_{\alpha}^{s} /\left(r_{0} d \theta\right)=g_{\alpha} / g, g=\sqrt{\left(g_{1}\right)^{2}+\left(g_{2}\right)^{2}}
$$

Therefore, the components of the normal outward to the boundary in Eqs (63), (64) are given as

$$
n_{1}=t_{2}, n_{2}=-t_{1}
$$

Finally, the weak form of the governing equations, Eqs (63), (64), can be written as

$$
\int_{0}^{2 \pi}\left[M_{\alpha \beta}^{*}+M_{\alpha \beta}^{m}-x_{\alpha}\left(Q_{\beta}^{*}+Q_{\beta}^{m}\right)\right] n_{\beta} J^{\prime} d \theta=q_{0} \bar{x}_{\alpha}^{i} \Omega_{s}^{i}, \int_{0}^{2 \pi}\left(Q_{\alpha}^{*}+Q_{\alpha}^{m}\right) n_{\alpha} J^{\prime} d \theta=q_{0} \Omega_{s}^{i},
$$

in which the bending stiffness $D$ is variable along the boundary $\Gamma_{s}^{i}$. Considering Eqs (15), (16) for DDM and Eq. (65) for $M_{\alpha \beta}^{m}, Q_{\alpha}^{m}$ together with meshless polynomial interpolation, we obtained the linear algebraic equations from Eq. (72) in terms of the coefficients of the Chebyshev polynomials and nodal displacements. The meshless approach is applicable also to the homogeneous plate. Then, the fundamental solutions of the moments and shear forces in Eq. (72) disappear and the weak form governing equations become

$$
\int_{0}^{2 \pi}\left(M_{\alpha \beta}^{m}-x_{\alpha} Q_{\beta}^{m}\right) n_{\beta} J^{\prime} d \theta=q_{0} \bar{x}_{\alpha}^{i} \Omega_{s}^{i}, \int_{0}^{2 \pi} Q_{\alpha}^{m} n_{\alpha} J^{\prime} d \theta=q_{0} \Omega_{s}^{i}
$$


where $M_{\alpha \beta}^{m}$ and $Q_{\alpha}^{m}$ represent now the whole bending stress-couples and shear stress-resultants respectively. Because all integral functions in Eqs (72) and (73) are regular in the domain except the crack surface, hence, any standard integral algorithm is valid. To employ the MLPG, the main advantage is that the first order partial derivatives of displacement $w_{k, \alpha}$ are required only in Eqs (72) and (73) in the computation process for homogenous and non-homogenous materials.

\section{Numerical examples}

In order to demonstrate the efficiency and accuracy of the hybrid MDDM with either PCM or MLPG, a rectangular plate of $2 H \times 2 W$ containing a central crack of length $2 a$ and a rectangular plate of $2 \mathrm{H} \times W$ containing an edge crack of length $a$ are under investigation. Both homogenous and non-homogenous materials are considered. Without specifications in each example, the default values are listed in Table 2. Nodal distributions in the normalized square domain $-1 \leq\left(\eta_{1}, \eta_{2}\right) \leq 1$ are chosen as

$$
\eta_{1}^{(i)}=\cos \frac{(i-1) \pi}{\left(L_{1}-1\right)}, \quad i=1,2, \ldots, L_{1} \text { and } \eta_{2}^{(j)}=\cos \frac{(j-1) \pi}{\left(L_{2}-1\right)}, j=1,2, \ldots, L_{2}
$$

and the dimensionless radius of the local integral domain in MLPG $r_{0}$ is chosen as $1 / 4\left(L_{1}-1\right)$. The numerical results show that the selection of the radius $r_{0}$ has very limited influence.

Table 2. Default setting

\begin{tabular}{lll}
\hline$E_{0}$ & 1 & Young's modulus \\
$v$ & 0.3 & Poisson Ratio \\
$h / a$ & 0.2 & Normalized thickness \\
$H / a$ & 2 & Normalized height \\
$W / a$ & 2 & Normalized width \\
$N_{c}$ & 10 & Chebyshev number \\
$L_{1}$ & 20 & Node number \\
$L_{2}$ & 20 & Node number \\
\hline
\end{tabular}




\section{Example 2. A simply supported square plate with a centre crack}

A simply supported square plate with a center crack of length $2 a$, height $2 H$ and width $2 W$ subjected to a uniformly distributed pressure load $q_{0}$ is considered first, as shown in Figure 7. Three different ratios of width and thickness are considered, i.e. $W / h=2,6$ and 10. This simplified panel in homogenous media was observed by Sosa and Eishen [42] and Dirgantara [11]. Figure 8 shows the normalized bending stress resultant intensity factors against the normalized crack length $a / W$. As it can be seen, the results by hybrid MDDM (MLPG) are in excellent agreement with those presented by Sosa and Eischen [42]. In addition, the difference between the results by the hybrid MDDM (PCM) and the dual BEM reported by Dirgantara [10] is very small. To show the convergence and accuracy of the hybrid MDDM, the values of the normalized bending stress resultant intensity factors for nodal density $\left(L_{m}\right)$ when $W / h=2$ and $a / W=0.5$ are found in Table 3. From Table 4, it is seen that, when $L_{1}=L_{2}>5$, the difference between the results of the PCM and MLPG is about 0.1 percent. Furthermore, we noticed that there is no difference between those results with different values of radius $r_{0}$ shown in Table 4 .

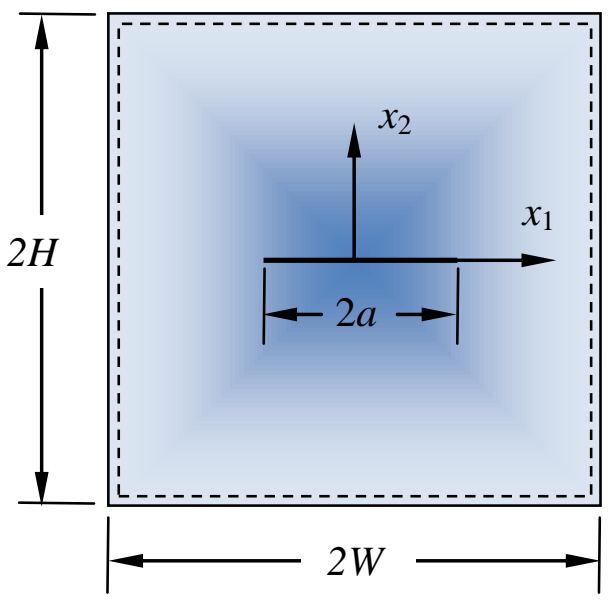

(a)

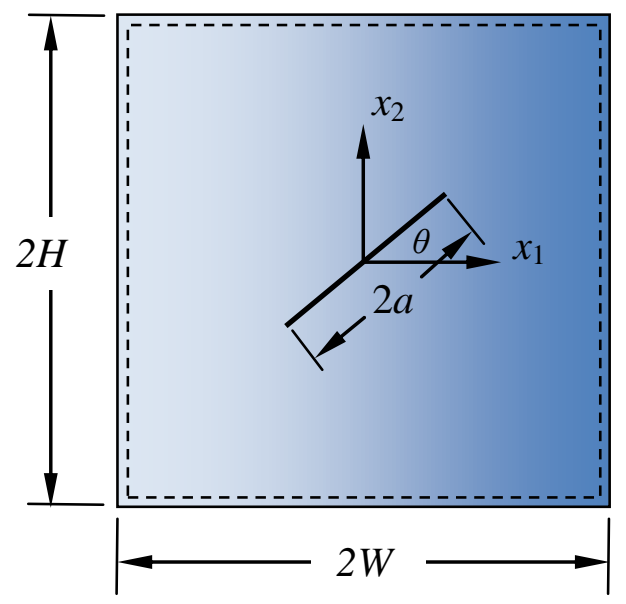

(b)

Figure 7. Simply supported square plate with center crack: (a) horizontal crack; (b) slant crack. 
Table 3. Normalized SIF $K_{I}^{b} /\left(q_{0} W^{2} \sqrt{\pi a}\right)$

\begin{tabular}{ccc}
\hline$L_{1}, L_{2}$ & PCM & MLPG \\
\hline 5,5 & 0.1174 & 0.1138 \\
11,11 & 0.1153 & 0.1116 \\
15,15 & 0.1143 & 0.1116 \\
\hline
\end{tabular}

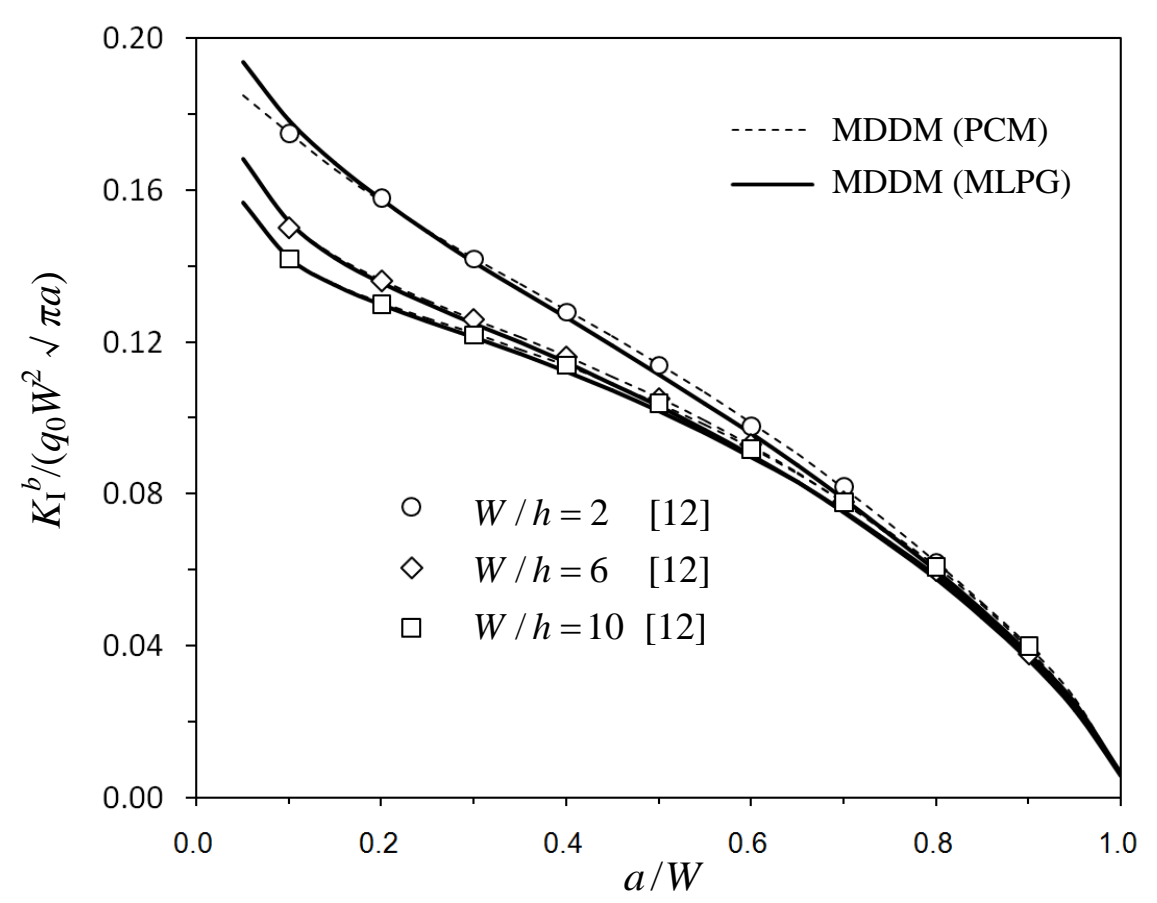

Figure 8. Normalized stress resultant intensity factor $K_{\mathrm{I}}^{b} / q_{0} W^{2} \sqrt{\pi a}$ under uniform pressure load $q_{0}$.

Table 4. Convergence investigation with local integral size

\begin{tabular}{lcccc}
\hline$r_{0} /(0.1 a)$ & 0.5 & 0.25 & 0.125 & 0.0625 \\
\hline$K_{I}^{b} /\left(q_{0} W^{2} \sqrt{\pi a}\right)$ & 0.1116 & 0.1116 & 0.1116 & 0.1116 \\
\hline
\end{tabular}

\section{Example 3. A square FGM plate with a centre crack}

The same configuration of the plate with a uniform pressure load $q_{0}$ as in Example 2, but non-homogenous media is being investigated now. Firstly, we consider a square plate with either four simply supported edges or clamped edges with normalized thickness $h / a=1 / 3$ and width $W / a=2$. Young's modulus is graded horizontally as $E=E_{0} f\left(x_{1}\right)$, where $f\left(x_{1}\right)=1+$ 
$\left(\alpha_{1}-1\right) x_{1}^{2} / W^{2}, \alpha_{1}=E_{w} / E_{0}$ and $E_{w}=E\left(x_{1}=W\right)$. The values of the normalized stress resultant intensity factors for various ratios $\alpha_{1}$ with different supports are given by the curves in Figures 9 and 10 respectively. In order to compare other numerical methods, the finite block method developed by Wen et al [36,37] is considered as reference. Due to the symmetry of the problem, a quarter plate is modeled with two blocks only. The COD technique and Singular Stress Method (SSM) are employed to calculate the stress resultant intensity factors. A good agreement can be observed between those approaches.

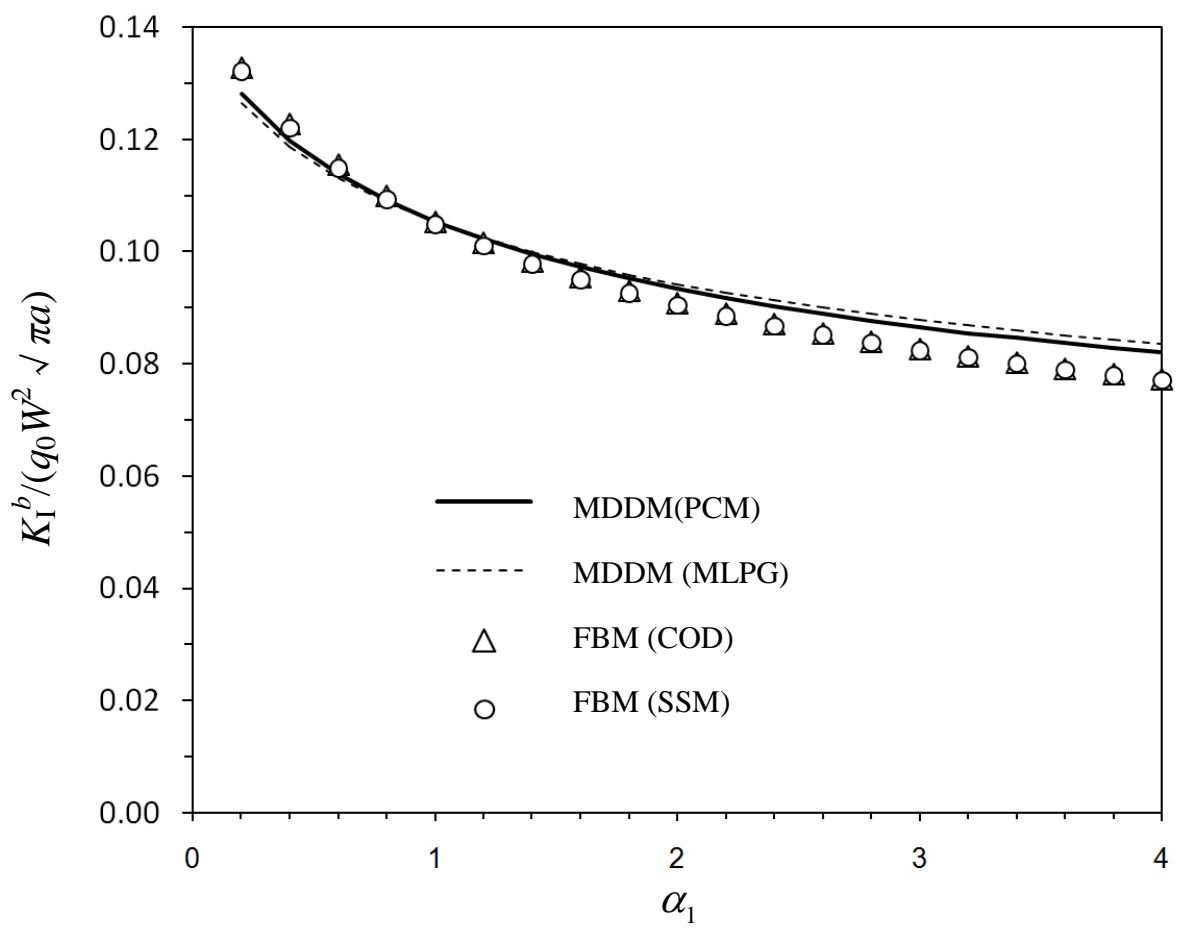

Figure 9. Normalized stress resultant intensity factors $K_{\mathrm{I}}^{b} /\left(q_{0} W^{2} \sqrt{\pi a}\right)$ for a simply supported plate subjected to uniform pressure load with $q_{0}$ by different approaches: MDDM (PCM): hybrid MDDM and point collocation; MDDM (MLPG): hybrid MDDM and meshless local Petro-Galerkin; FBM (COD): crack opening displacement; FBM (SSM): singular stress method.

Secondly, we consider Young's modulus varying with $E=E_{0} f\left(x_{1}, x_{2}\right)$, where $f\left(x_{1}, x_{2}\right)=$ $e^{\alpha_{1}\left|x_{1} / a\right| /+\alpha_{2}\left|x_{2} / a\right|}, \alpha_{1}=\ln \left(E_{W} / E_{0}\right)$ and $\alpha_{2}=\ln \left(E_{H} / E_{0}\right), E_{W}=E\left(x_{1}=W, x_{2}=0\right), E_{H}=E\left(x_{1}=0\right.$, $\left.x_{2}=H\right)$ and thickness $h / a=0.2 \sqrt{10}$. Normalized stress resultant intensity factors are given by 
the curves in Figures 11(a) and 11(b) respectively, versus the ratios $\alpha_{1}$ and $\alpha_{2}$ and different constraints. The comparisons with the finite block method using COD are shown in the same figures and the average relative error is in $3 \%$.

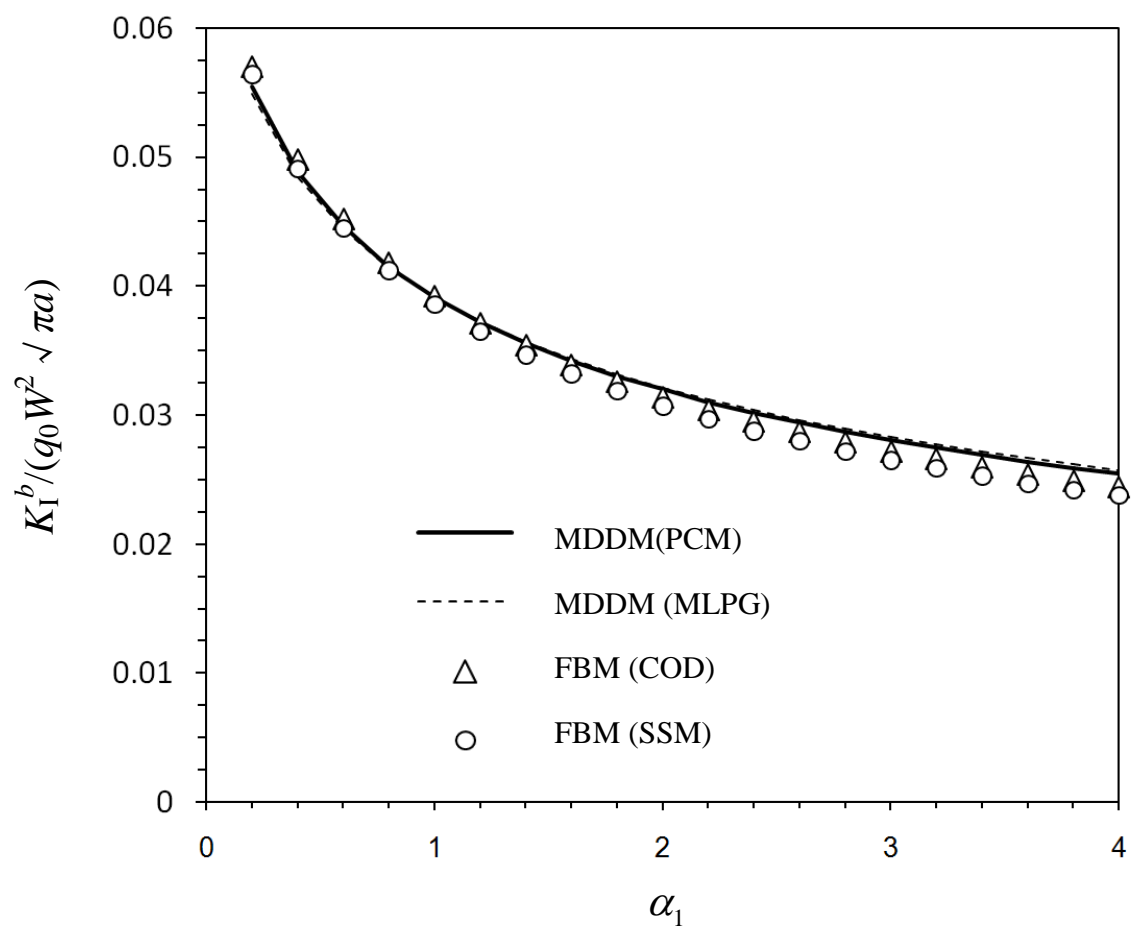

Figure 10. Normalized stress resultant intensity factors $K_{\mathrm{I}}^{b} /\left(q_{0} W^{2} \sqrt{\pi a}\right)$ for a clamped plate subjected to uniform pressure load with $q_{0}$ by different approaches.

Finally, a square plate with a slant center crack is analyzed. Young's modulus is graded as $E=E_{0} e^{\alpha_{1}\left|x_{1}\right| / a}$, where $\alpha_{1}=\ln \left(E_{W} / E_{0}\right)$ and thickness $h / a=0.2 \sqrt{10}$ and $W / a=2$. The variation of the normalized stress resultant intensity factors by the hybrid MDDM (MLPG) is shown in Figures 12(a) and 12(b) against the slant angle $\theta$ and $\alpha_{1}$. In addition, we noticed that the absolute values of mode II and mode III stress resultant intensity factors are too small to be presented. 

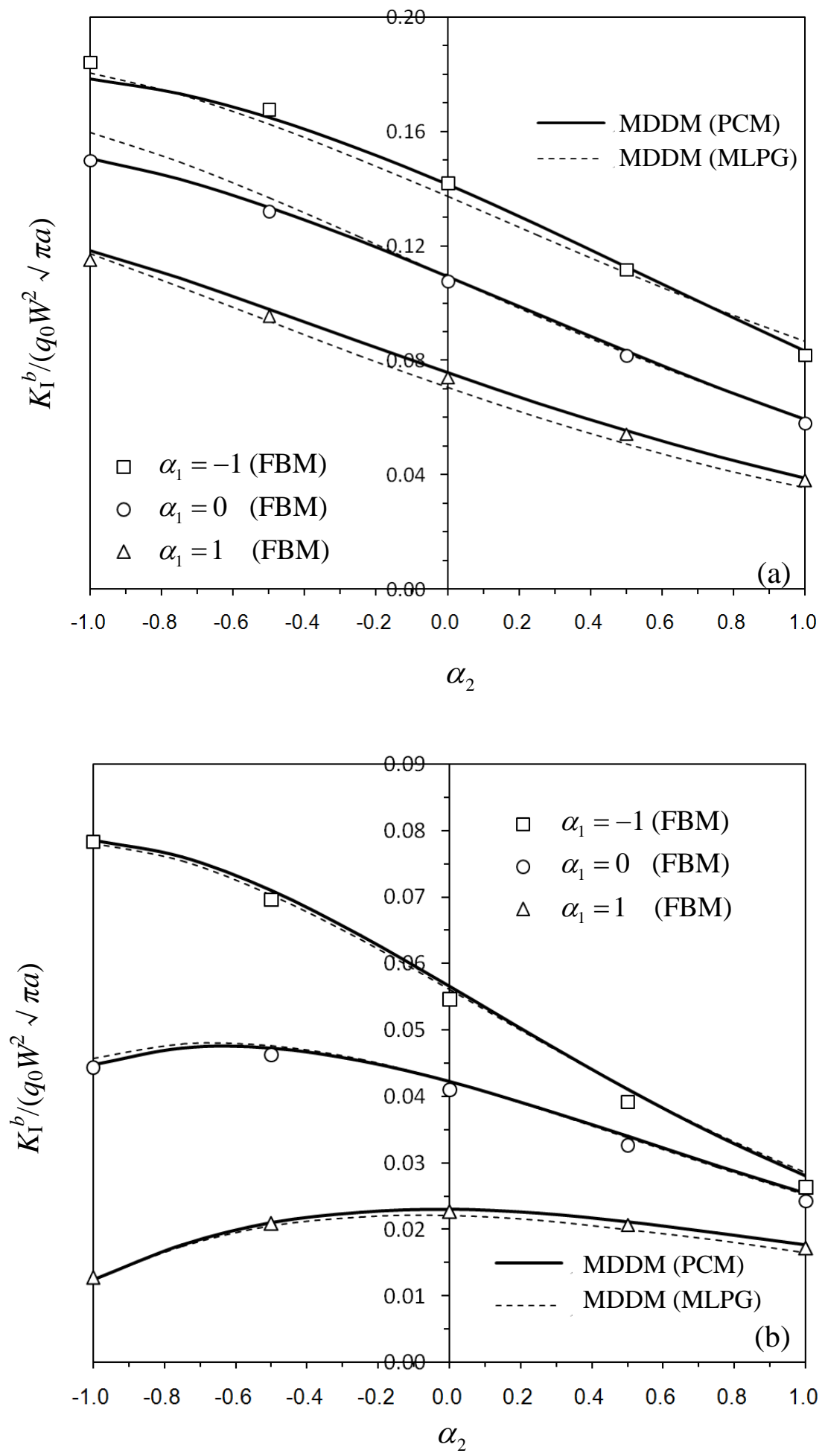

Figure 11. Normalized stress resultant intensity factor $K_{\mathrm{I}}^{b} / q_{0} W^{2} \sqrt{\pi a}$ subjected to uniform pressure load: (a) simply supported edges; (b) clamped edges. 

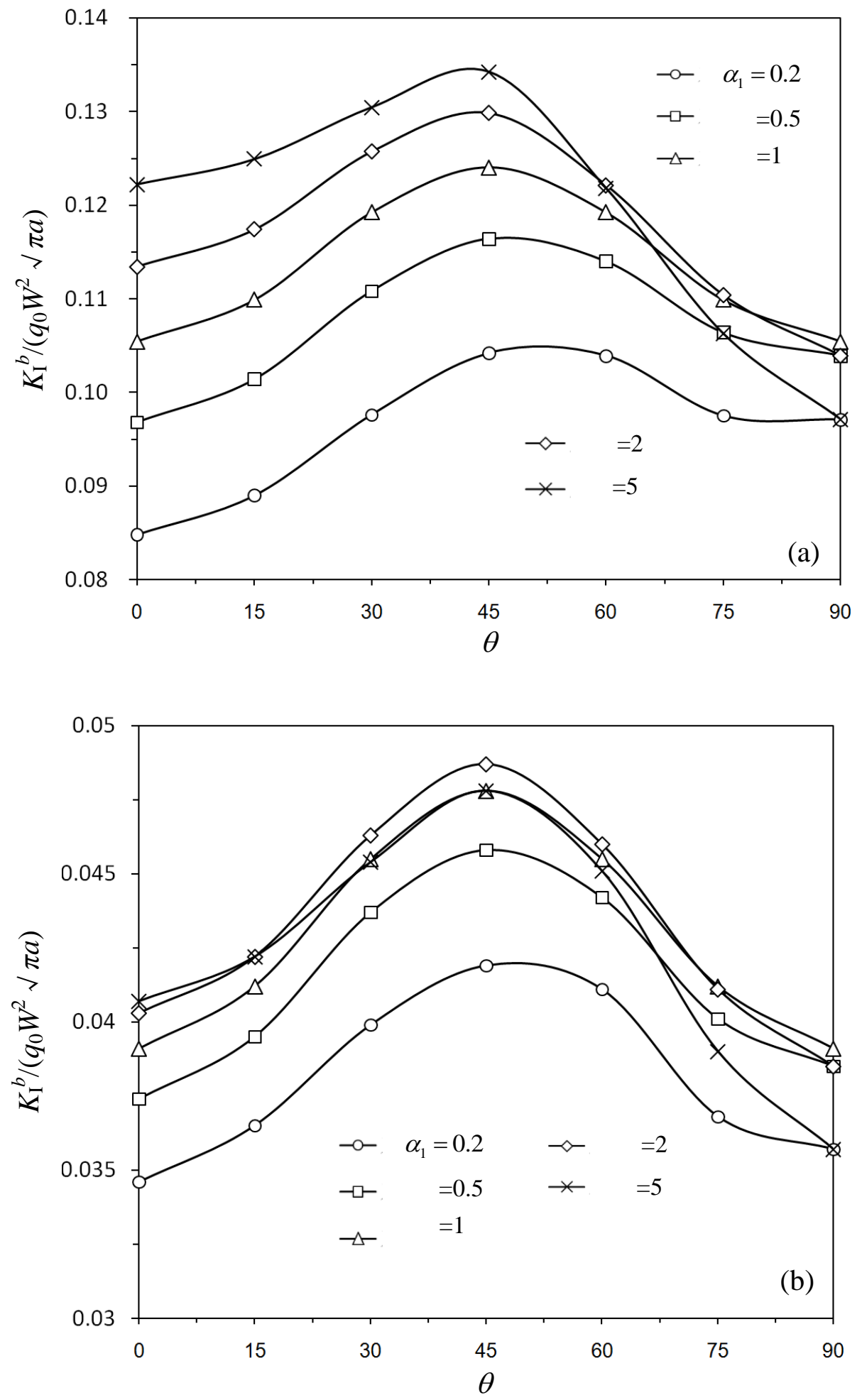

Figure 12. Normalized stress resultant intensity factor $K_{\mathrm{I}}^{b} / q_{0} W^{2} \sqrt{\pi a}$ with slant centre crack subjected to uniform pressure load: (a) simply supported edges; (b) clamped edges. 


\section{Example 4. A rectangular FGM plate with an edge crack}

Consider a rectangular plate of width $W$ and height $2 H$ containing an edge crack of length $a$ under uniform pressure load $q_{0}$, as shown in Figure 13, and ratios $H / W=1, h / W=0.1$. Young's modulus is graded as $E=E_{0} f\left(x_{1}\right)$, where $f\left(x_{1}\right)=e^{\alpha_{1} x_{1} / W}, \alpha_{1}=\ln \left(E_{w} / E_{0}\right)$ and $E_{w}=E$ $\left(x_{1}=W\right)$. We have the results of the normalized stress resultant intensity factors by MDDM (MLPG) shown in Figure 14 against the crack length $a / W$ and material parameter $\alpha_{1}$. Again, the results by the finite block method are presented in Figure 15 for comparison. Seeing from Figure 15, we found that the results by MDDM (MLPG) and FBM are in a good agreement with two blocks used in FBM. The error is bigger if the crack length is too small $(a / W \leq 0.1)$ or is too large $(a / W \geq 0.9)$.

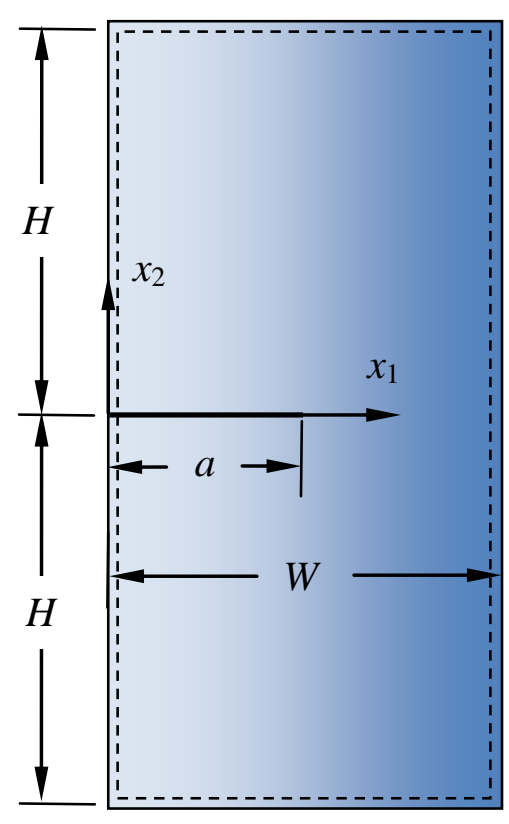

Figure 13. Rectangular plate with edge crack. 


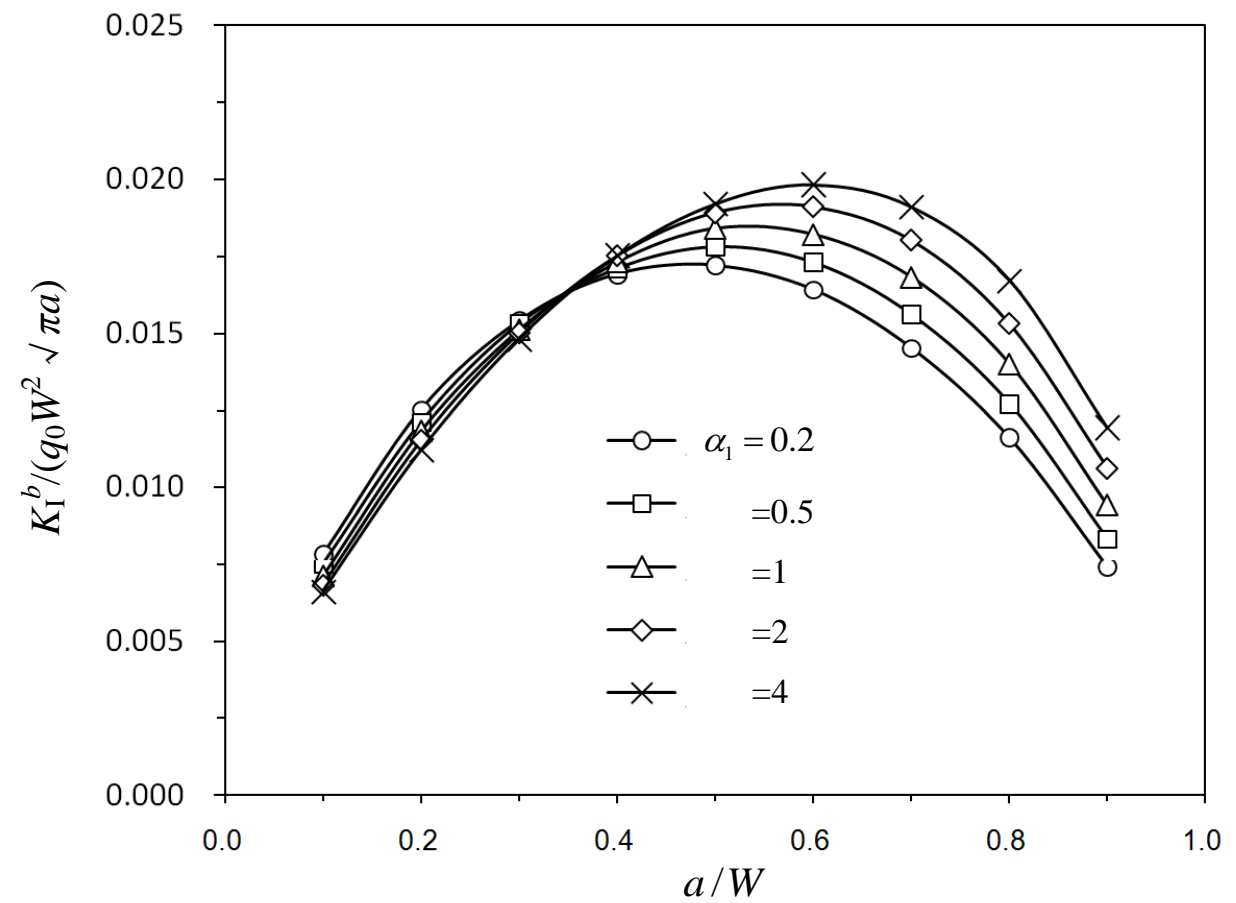

Figure 14. Normalized stress resultant intensity factor $K_{\mathrm{I}}^{b} / q_{0} W^{2} \sqrt{\pi a}$ with four simply supported edges.

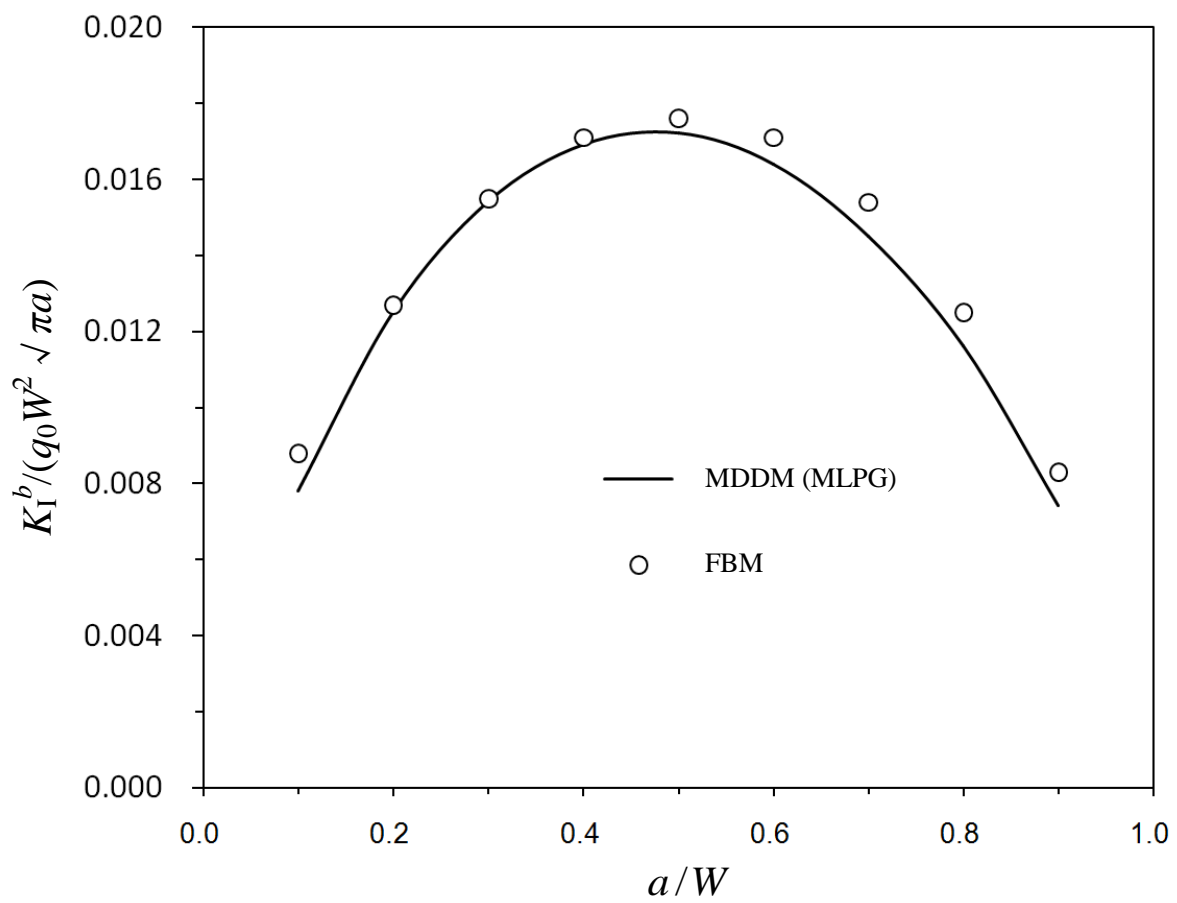

Figure 15. Comparison with the results by FBM when $\alpha_{1}=0.25$. 


\section{Conclusion}

A hybrid meshless displacement discontinuity method is formulated to the bending of the FGM Reissner's plate with cracks in this paper. It is the first attempt to solve the crack problems in the continuous non-homogeneous plate structures. The key point is that the fundamental solution for a homogenous body is employed to generate the displacement discontinuity on the crack surface. In the MLPG, a unit step function is utilized as the test function in the local weak-form of governing equations with Lagrange polynomials interpolation. The advantages of the boundary element method and meshless approaches are inherited in the hybrid MDDM, to deal with the fracture problems. By introducing the Chebyshev polynomials in the hypersingular integral equations, the analytical solution of the stress intensity factors can be obtained with high accuracy. Several numerical examples demonstrate the accuracy and efficiency of the present hybrid meshless displacement discontinuity method via tests in the case of homogeneous media. The following is the summary of the hybrid meshless displacement discontinuity method applied to Reissner's plate(1) The fundamental solutions found in the case of homogenous media are useful also for non-homogenous media;

(2) Highly accurate solution can be obtained by the meshless approaches, as all components of stress and displacement are continuous on the interface between each two blocks;

(3) Numerical solutions exhibit convergence with increasing the number of the Chebyshev polynomials in DDM as well as the node density used by MLPG;

(4) Crack propagations can be solved easily without re-meshing.

\section{Acknowledgment.}

The authors acknowledge the support of the Slovak Science and Technology Assistance Agency registered under number APVV-18-0004, VEGA-2/0061/20 and the National Natural Science Foundation of China (Grant-no:11702125). 


\section{References}

[1] Zienkiewicz, O.C. and Taylor, R. The Finite Element Method,, Vol 2: Non Linear Problems, 4th edition, McGraw-Hill Publishing Company, London, 1994.

[2] Barsoum, R.S. A degenerate solid element for linear fracture analysis of plate bending and general shells, Int. J. for Numerical Methods in Eng., 10, 551-564, 1976.

[3] Barsoum, R.S., Loomis, R. W. and Stewart, B.D. Analysis of through crack in cylindrical shells by quarter point elements, Int. J. Fract., 15(3), 259-280, 1979.

[4] Ehlers, R. Stress intensity factors and crack opening areas for axial through cracks in hollow cylinders under internsl pressure loading, Eng. Frat.Mech., 25(1), 63-77,1986.

[5] Jaswon, M.A., Maiti, M. and Symm, G.T. Numerical biharmonic analysis and some applications, Int. J. Solids and Struct., 3, 309-332, 1967.

[6] Vander Weeën, F. Application of the boundary integral equation method to Reissner's plate model, Int. J. for Numerical Methods in Eng., 18, 1-10, 1982.

[7] Karam, V.J. and Telles, J.C.F. On the boundary elements for Reisner's pate theory, Engineering Analysis, 5, 21-27, 1988.

[8] Rashed, Y.F., Aliabadi, M.H. and Brebbia, C.A. Hyper-singular boundary element formulation for Reissner plates, Int. J. Solids and Struct., 35(18), 2229-2249, 1998

[9] Rashed, Y.F., Aliabadi, M.H. and Brebbia, C.A. Stress and displacement boundary integral formulations for shear deformable plate bending analysis, in Boundary Element Methods XVIII, C.A Brebbia, J.B. Martins, M.H. Aliabadi (Eds), Portugal, 493-502, Computational Mechanics Publication, 1996.

[10] Dirgantara, T. and Aliabadi, M.H. A new boundary element formulation for shear deformable shells analysis, Int. J. for Numerical Methods in Eng., 45, 1257-1275, 1999

[11] Dirgantara, T. and Aliabadi, M.H. Crack growth analysis of plate loaded bending and tension using dual boundary element method, Int. J. Fract., 105(1), 27-47, 2000.

[12] Dirgantara, T. and Aliabadi, M.H. Dual boundary element formulation for fracture mechanics analysis of shear deformable shells, Int. J. Solids and Struct., 38, 7769-7800, 2000.

[13] Wen, P.H., Aliabadi, M.H. and Young, A. Fracture mechanics analysis of curved stiffened panels using BEM, Int J. Solids and Structures, 40, 219-236, 2003. 
[14] Wen, P.H., Aliabadi, M.H. and Young, A. Crack growth analysis for multi-layered airframe structures by boundary element method, Eng. Fract. Mech., 71, 619-631 (2004).

[15] Wen, P.H., Aliabadi, M.H. A. Large deformation analysis of Reissner plate by boundary element method, Computers and Structure, 83, 870-879 (2005).

[16] Aliabadi, M.H. The Boundary Element Method Vol2: Applications in Solids and Structures, John Wiley and Sons, 2002.

[17] Nayroles, B., Touzot, G. andVillon, P. Generalizing the finite element method: diffuse approximation and diffuse elements, Computational Mechanics, 10, 307-318, 1992.

[18] Belytschko, T., Lu, Y.Y. \& Gu L. Element-free Galerkin method, Int. J. Numerical Methods in Engineering, 37, 229-256, 1994.

[19] Liu, W.K., Jun, S. \& Zhang Y. Reproducing kernel particle methods, Int. J. Numerical Methods in Engineering, 20, 1081-1106, 1995.

[20] Atluri, S.N. \& Zhu, T. A new meshless local Peyrov-Galerkin (MLPG) approach to nonlinear problems in computational modelling and simulation, Comput Model Simul Engng, 3, 187-196, 1998.

[21] Atluri, S.N. \& Zhu, T. A new meshless local Peyrov-Galerkin (MLPG) approach in computational mechanics, Comput Mech, 22, 117-127, 1998.

[22] Atluri, S.N. The Meshless Method (MLPG) for Domain and BIE Discretizations, Forsyth, GA, USA, Tech Science Press, 2004.

[23] Sladek, J., Sladek, V. \& Zhang, Ch. Heat conduction analysis in nonhomogeneous anisotropic solid, ZH Yao, MW Yuan, WX Zhong edited, Computational Mechanics, Tsinghua University Press and Springer, 609-614, 2004.

[24] Sladek, J. Sladek, V \& Atluri, S.N. Meshless Local Petrov-Galerkin method for heat conduction problem in an anisotropic medium, Comput Model Engng Sci, 6, 309-318, 2004.

[25] Sladek, V., Sladek, J., Tanaka, M. \& Zhang, Ch. Local integral equation method for potential problems in functionally graded anisotropic materials, Engng Analysis with Boundary Elements, 29, 829-843, 2005.

[26] Liu, G.R. Meshfree Methods: Moving Beyond the Finite Element Method, Second Edition, CRC Press, Taylor and Francis Group, New York, 2010. 
[27] Guimarães, S. Telles JCF. The method of fundamental solutions for fracture mechanics Reissner's plate application, Engng Analysis with BE, 33, 1152-1160, 2009.

[28] Guimarães, S. Telles JCF. On the numerical Green's function technique for cracks in Reissner's plates, Computer Methods in Applied Mechanics and Engng, 196, 2478-2485, 2007

[29] Sladek, J., Sladek, V., Krivacek, J., Wen, P.H., Zhang Ch. Meshless Local Petrov-Galerkin (MLPG) method for Reissner-Mindlin plates under dynamic load, Computer Methods in Applied Mechanics and Engineering, 196, 2681-2691, 2007.

[30] Sladek, J., Sladek, V., Solek, P., Wen, P.H. Thermal bending of Reissner-Mindlin plates by the MLPG, CMES-Computer Modeling in Engineering and Sciences, 28(1), 57-76, 2008.

[31] Sladek, J., Sladek, V., Solek, P., Wen, P.H., Atluri, S.N. Thermal analysis of ReissnerMindlin shallow shells with FGM properties by the MLPG, CMES-Computer Modeling in Engineering and Sciences, 30(2), 2008, 77-97.

[32] Wen, P.H., Hon, Y.C. Geometrically nonlinear analysis of Reissner-Mindlin plate by meshless computation, CMES: Computer Modeling in Engineering\& Sciences, 21(3), 2007, 177-191.

[33] Portela, A., Aliabadi, M.H. and Rooke, D.P. Dual boundary incremental analysis of crack propagation, Computer and Structures 1993; 46 (2): 237-247.

[34] Mi Y. and Aliabadi, M.H. Three-dimensional crack growth simulation using BEM, Computers and Structures 1994; 52: 871-878.

[35] Crouch S.L. and Starfield, A.M. Boundary Elements in Solid Mechanics, George Allen and Unwin, London, 1983.

[36] Wen, P.H. The calculating stress intensity factor for a rigid wedge inserted into a halfinfinite crack in an orthotropic plate. Chinese Journal of Applied Mechanics 1998; 5(4): 8996.

[37] Wen, P.H. The solution of a displacement discontinuity for an anisotropic half-plane and its applications to fracture mechanics, Engineering Fracture Mechanics 1986; 35(5/6): 1145-1154.

[38] Li, J., Sladek, J., Sladek, V. and Wen, P.H. Hybrid meshless displacement discontinuity method (MDDM) in fracture mechanics: static and dynamic, European Journal of Mechanics /A Solids, 2020 (accepted for publication). 
[39] Reissner, E. On a variational theorem in elasticity, J. Mathematics and Physics, 29, 90-95, 1950.

[40] Kaya, A.C. and Erdogan, F. On the solution of integral equations with strongly singular kernels, Quarterly of Applied Mathematics, 45(1), 105-122, 1987.

[41] Sih, G.C. Handbook of Stress Intensity Factors, Lehigh University, Institute of Fracture and Solid Mechanics, 1973.

[42] Sosa, H.A. and Eischen, J.W. Computation of stress intensity factors for plate bending vis a path of independent integral, Eng. Frat. Mech., 25,451-262, 1986.

[43] Wen, P.H., Cao P. and Korakianitis, T. Finite Block Method in elasticity. Engineering Analysis With Boundary Elements, 46, 116-125 (2014).

[44] Li, M and Wen, P.H. Finite block method for transient heat conduction analysis in functionally graded media. Int. J. For Numer. Methods in Eng., 99(5) 372-390 (2014).

[45] Sladek, V., Sladek, J., Zhang, Ch. Local integro-differential equations with domain elements for the numerical solution of partial differential equations with variable coefficients. Journal of Engineering Mathematics, 51, 261-282 (2005).

[46] Sladek, V., Sladek, J., Zhang, Ch. Domain element local integral equation method for potential problems in anisotropic and functionally graded materials. Computational Mechanics, 37, 78-85 (2005).

[47] Sladek, V., Sladek, J., Zhang, Ch. The use of finite elements for approximation of field variables on local sub-domains in a mesh-free way, Chapter 6 in: Composites with Micro and-Nano-Structure (V. Kompis, ed.), Springer Science, Heidelberg, 2008, pp. 87-106. 